
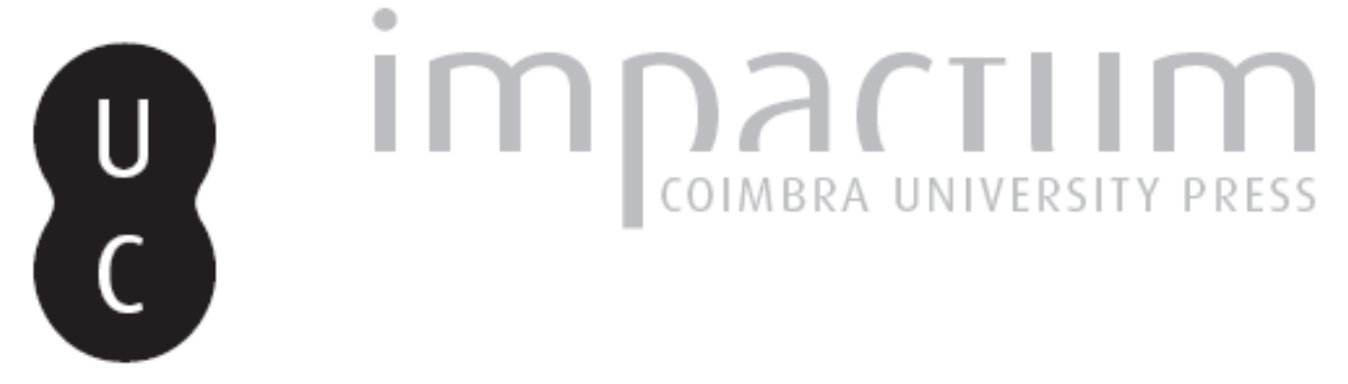

L' Erotikos: un éloge du Dieu Éros? Une relecture du dialogue de Plutarque

Autor(es): $\quad$ Frazier, Françoise

Publicado por: International Plutarch Society

URL persistente:

URI:http://hdl.handle.net/10316.2/37608

DOI:

DOI:http://dx.doi.org/10.14195/0258-655X_3_4

Accessed : $\quad$ 26-Apr-2023 15:18:23

A navegação consulta e descarregamento dos títulos inseridos nas Bibliotecas Digitais UC Digitalis, UC Pombalina e UC Impactum, pressupõem a aceitação plena e sem reservas dos Termos e Condições de Uso destas Bibliotecas Digitais, disponíveis em https://digitalis.uc.pt/pt-pt/termos.

Conforme exposto nos referidos Termos e Condições de Uso, o descarregamento de títulos de acesso restrito requer uma licença válida de autorização devendo o utilizador aceder ao(s) documento(s) a partir de um endereço de IP da instituição detentora da supramencionada licença.

Ao utilizador é apenas permitido o descarregamento para uso pessoal, pelo que o emprego do(s) título(s) descarregado(s) para outro fim, designadamente comercial, carece de autorização do respetivo autor ou editor da obra.

Na medida em que todas as obras da UC Digitalis se encontram protegidas pelo Código do Direito de Autor e Direitos Conexos e demais legislação aplicável, toda a cópia, parcial ou total, deste documento, nos casos em que é legalmente admitida, deverá conter ou fazer-se acompanhar por este aviso.

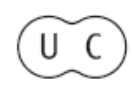




\section{PLOUTARCHOS, n.s.}

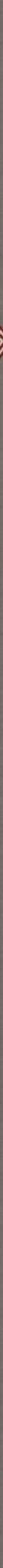

UNIVERSITY OF MÁLAGA (SPAIN)

UtAH STATE UNIVERSity, LOGAN, UTAH (U.S.A.) 


\title{
L'Erotikos : un éloge du Dieu Éros? Une relecture du dialogue de Plutarque ${ }^{1}$ par \\ Françoise Frazier \\ Université de Montpellier III
}

\begin{abstract}
This article reconsiders the unity and coherence of Plutarch's Erotikos, a highly debated theme, in order to bring a light over the philosophical dimension of the dialogue. A thorough examination of the composition shows at first how logoi and praxis (the affair of Ismenodora and Bacchon) are skilfully interweaved and how they bind the themes of love and marriage. Then the logoi themselves can be divided into three dif ferent parts ; in each of them Plutarch deals with the same themes and similes, borrowed especially from Plato's Phaedrus, and brings out the divinity, the power and the bene volence of the God Eros - these three qualities are topoi of eulogy and remind the rea der even more of Plato's Symposion. Hence, I finally suggest that the whole work could be read as a huge eulogy, in which Plutarch celebrates the essential part Eros plays in religious tradition as well as in philosophy, in human life as well as after death.
\end{abstract}

Eh bien, allons, par Zeus, allons nous moquer de notre homme et adorer le Dieu, car, à l'évidence, il se réjouit et assiste avec bien veillance ce qui est en train de s'ac complir (26.771 D-E).

Ces paroles de Plutarque, qui conclu cation du cortège nuptial d'Isménodore et de Bacchon, reflètent bien la tonalité particulière du texte, ou plutôt la multi plicité de ses tons : ton de moquerie lé gère à l'égard du rival malheureux d'Isménodore, qui s'accorde avec le caractère paradoxal de la prophasis, trai tée dans un style proche du théâtre d'un côté ${ }^{2}$, grande révérence pour le Dieu

1 Cet article a déjà fait l'objet d'une première publication, destinée aux candidats à l'a grégation, dans Y Information littéraire 1998. Je souhaitais proposer mon interprétation aux spécialistes de Plutarque et je remercie Aurelio Pérez Jiménez d'avoir accepté d'en publier une version légèrement remaniée dans Ploutarchos.

2 Le texte est jalonné de remarques rapprochant l'histoire d'Isménodore et de Bacchon du théâtre : ainsi d'entrée, le narrateur Autoboulos présente le 'prétexte' à la conversation

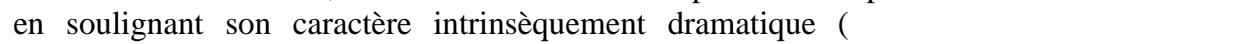

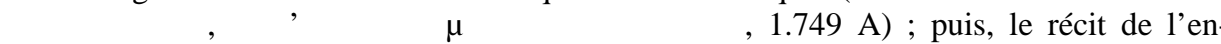
lèvement y insiste encore par les réactions, tant à Thespies, où l'on déserte le théâtre 
présenté comme partie prenante dans l'histoire après avoir été l'unique sujet des logoi ${ }^{3}$. Les citer, c'est ainsi poser le problème, crucial pour l'interprétation du dialogue, de son unité, tenter de dépasser une apparente diversité, d'au tant plus embarrassante que le Banquet de Platon, sans cesse invoqué comme modèle par les critiques même s'ils re connaissent que le Phèdre joue un rôle encore plus important, possède une forte unité, avec un discours de Diotime qui reprend et dépasse les éléments de véri té épars dans les éloges précédents et un discours d'Alcibiade qui suggère l'iden tification entre cet amour philosophe et la figure atopos de Socrate ${ }^{4}$. La recher che de l'unité et du sens de l'œuvre se trouve ainsi liée à la question de la fidé lité à Platon : on sait que R. Hirzel le trouvait à ce point trahi qu'il refusait la paternité du dialogue à Plutarque5; mais on sait aussi que K. Ziegler proclamait ne rien connaître de "plus authentique ment plutarquéen" que ce texte 6 . Plus récemment, J. M. Rist a rappelé très jus tement les orientations propres à Plutar que et à son époque, où la philosophie se veut maîtresse de vie, et la nécessité d'une réactualisation de Platon, qui sup pose la confrontation avec les autres écoles. Mais, ces principes posés, le spécialiste de philosophie qu'il est pro pose d'unifier le texte autour de la polé mique antiépicurienne et s'en tient à un examen analytique des questions philo-

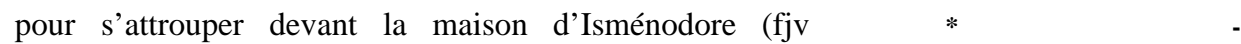

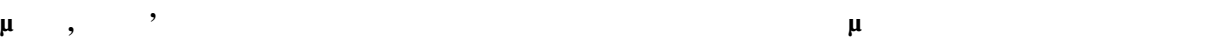

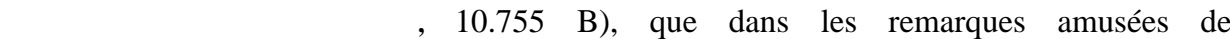

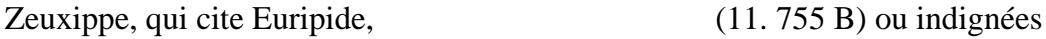
de Protogène, qui s'enflamme sur un ton tragique ( “ " et termine dans un style plus proche d'Aristophane : ï $\omega \varepsilon \boldsymbol{\varepsilon} \dot{\eta} \varepsilon \hat{\imath}, \quad i \omega \varepsilon v, \varepsilon i \pi \varepsilon v, \dot{0} \pi \omega$

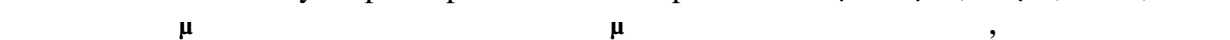

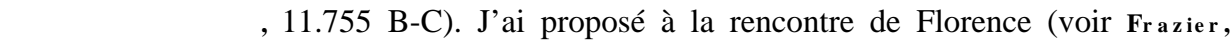
2005b) une interprétation de cette présentation, qui n'engage pas à mon avis la structure d'en semble de l'œuvre et n'autorise pas à parler de 'dialogue dramatique', sauf à entendre par là l'intrication de logoi et de pragmata que j'analyserai infra.

Il faut noter la dernière expression : le Dieu, dont on a parlé, assiste aussi тoî $\pi \rho \alpha \tau \tau$ غ่vor .

$4 \quad$ L'article sur lequel je me fonde essentiellement est celui de Ba but , 1982.

Hirzel, 1895, t. II, 233-234 ; même jugement chez Russe11, 1973, 92, qui évoque « an anti-Platonic, almost anti-philosophical cause. »

6 R.E. 21.1, 1951, col. 796, "Nichts echter Plutarchisches làBt sich erdenken als dieser Dialog."

7 Rist, 2001, 559 : “The Amatorius, then, offers a 'Platonic' évaluation of human expé riences available to most of us, not just to the self-conscious followers of the Diotima of the Symposium or to the philosophical lovers and kings of the Republic." 
sophiques que la tradition platonicienne léguerait à Plutarque ${ }^{8}$. Je voudrais pour ma part prendre en compte, dans un esprit qui me semble fidèle à la tradition platonicienne, à la fois la facture littérai re et le contenu philosophique pour met tre en lumière le sens de ce texte foison nant : ce qui exige au préalable de défi nir nettement les composantes de l'œu vre et de déterminer où et en quoi leur unification semble problématique; ce n'est que dans un second temps qu'on peut essayer de dépasser cette diversité, indéniable, pour dégager les grandes lignes de force qui réaffirment d'une partie à l'autre la divinité et la puissance bienfaisante d'Éros et proposer de lire

L'Érotikos comme un éloge du Dieu.

\section{Une unité problématique}

Les éléments formels : logoi et praxis

Le premier élément évident de diver sité, et le plus facile à réduire, réside dans l'entrelacement de l'action et du discours. C'est un style cher à Plutarque et qu'il maitrise bien : on le trouve dans le De Pythiae oraculis, où la conversa tion épouse la visite de Delphes du jeune Diogénianos ${ }^{9}$, et plus encore dans le $D e$ Genio Socratis, où des conversations philosophiques variées s'insèrent dans le récit des dernières heures vécues par les conjurés avant la libération de la Cadmée sans qu'on réussisse d'emblée à démêler, dans ce jeu compliqué, quel est le rapport exact de l'action et de la dis cussion $^{10}$. Ici, au contraire, il existe une liaison intime bien visible, dont L. Goessler a dégagé les éléments essen tiels $^{11}$ : non seulement, selon les termes d'Autoboulos, l'histoire est "l'occasion dont a jailli la discussion ${ }^{12 "}$ "et, après avoir évoqué le petit groupe d'amis qui s'est retiré sur l'Hélicon pour philoso pher à loisir (2.749 B-C), le narrateur introduit immédiatement Pisias et Anthémion, qui viennent "dès l'aube" sou mettre à l'arbitrage de Plutarque et de ses amis le sort du jeune Bacchon, recherché en mariage par une jeune et riche veuve, Isménodore, amie de sa mère (2.749 C-F), mais encore, après les premiers échanges sur ce sujet, l'annon ce de l'enlèvement du jeune homme per met à la discussion de prendre toute son ampleur : les principaux intéressés s'en

8

Voir le compte-rendu de son article dans Ploutarchos, 2 (2004/2005), p. 172.

$9 \quad$ J'ai proposé une analyse au colloque Interpreting Composition in Plutarchos (Louvain 5-7 juillet 2001), "Delphes dans tout son éclat. Le De Pythiae oraculis couronnement des Dialogues Pythiques" (Actes à paraître).

10

Parmi les nombreuses tentatives, l'analyse de Babut, 1984, qu'on l'accepte ou non, témoigne à tout le moins de la difficulté à dégager une unité qui n'apparaît pas avec évi dence à la lecture, ce que confirme encore la multiplicité des interprétations proposées.

11

12 Go e ssl e r, 1962, chapitre 1, 22-29 ("Kompositionsanalyse”).

$\dot{\eta} \pi \rho \dot{\varphi} \varphi \alpha \sigma 1, \dot{\varepsilon} \xi \dot{\eta} \dot{\omega} \rho \dot{\eta} \theta \eta \sigma \alpha \nu$ oi $\lambda$ óyol... (1.749 A). 
vont pour essayer d'infléchir, dans un sens ou dans l'autre, les événements ${ }^{13}$ et le sérieux Pemptidès, qui n'avait rien dit jusque là, peut alors élargir la question et passer du cas particulier à l'Amour en général $(12.755 \mathrm{~F})$. À nouveau, l'histoi re est à l'origine de cet élargissement, puisque c'est l'interprétation de l'audace inouïe d'Isménodore comme le fruit d'une "inspiration divine, plus forte que le raisonnement humain ${ }^{14}$ " qui le fait réagir et blâmer la divinisation indue d'une passion qu'il faudrait plutôt chas ser et réprimer; de là une longue inter vention de Plutarque, qui occupe les chapitres 13 à 20 et dont la fin a disparu dans une lacune ${ }^{15}$. Rien ensuite dans les chapitres 21-25, où il répond aux objec tions de Zeuxippe, elles aussi perdues, n'évoque le cas précis du couple thespien et c'est seulement après la fin des conversations ${ }^{16}$ que le récit reprend avec le retour à Thespies : comme ils ignorent visiblement comment les cho ses ont tourné17, on ne peut même pas affirmer qu'un messager était venu les chercher, liant ainsi leur retour à l'ac tion. Il ressort en tout cas du début du chapitre 26 que la dernière intervention de Plutarque s'est faite sur le chemin du retour et ce dernier chapitre consacre le triomphe de l'amour : L'Érotikos se con clut avec la formation du cortège nuptial ou, plutôt, le ralliement des participants

Les adversaires d'Isménodore, Pisias et Protogène, s'en vont les premiers, d'eux-mêmes (11.755 C), tandis qu'un messager vient, un peu plus tard, chercher son partisan, Anthémion (13.756 A). Go e ssi e r , 1962, a certainement raison de suggérer que ces départs permettent de ne plus avoir de participants partisans plutôt que d'imaginer avec Fl a c el iè r e, 1980,35, qu'u ne telle mise en scène rendrait crédible le dénouement. On peut cependant relever que l'avo cat d'Anthémion, Daphnée, si épris de Lysandra, ne s'en va pas, ce qui crée un déséquilibre léger en faveur de l'amour conjugal. En outre le décalage dans les départs permet à Anthémion de rester le temps d'attribuer à une "inspiration divine"cet acte d'audace insolite.

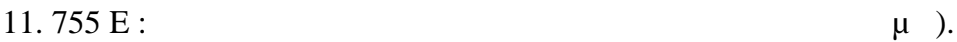
Sur cette lacune, voir Fl a c el ièr e, 1980, 42.

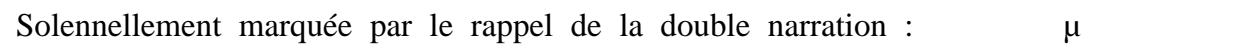

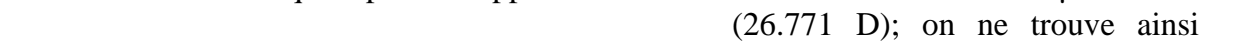
$\pi \alpha \tau \dot{\rho} \rho \varepsilon \varphi \eta$ qu'en trois occasions, à la première intervention de Plutarque $(6.752 \mathrm{C})$, pour annoncer l'arrivée du messager $(10.754 \mathrm{E})$ et pour introduire la réaction de Zeuxippe à la nouvelle de l'enlèvement (11.755 B); dans les deux premiers cas, on a un moment impor tant, pour la discussion ou pour l'action; dans le dernier, ce rappel sert peut-être à distancier un peu plus le fait, participant ainsi à la 'dédramatisation' de l'enlèvement.

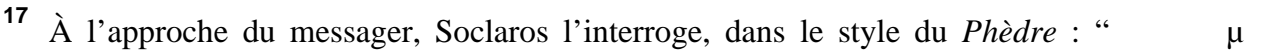
$\gamma^{\prime} \dot{\omega}$ ió$\gamma \varepsilon v \varepsilon$, $\dot{\alpha} \pi \alpha \gamma \gamma \varepsilon \dot{\varepsilon} \lambda \omega \nu$ " (26. $\left.771 \mathrm{D}\right)$; il ignore donc la 'conversion' de Pisias ; on peut imaginer au contraire qu'on est venu les chercher en leur disant que tout allait mal, ce qui rendrait le retournement voulu par le dieu encore plus spectaculaire, mais cette hypothèse est pure spéculation. 
au dialogue à une fête déjà commencée ${ }^{18}$. Le texte se referme ainsi sur luimême ${ }^{19}$ en une sorte de Ringskomposition $^{20}$, où la praxis forme comme un anneau enchâssant les logoi.

Les deux thèmes à articuler : mariage et amour

Parfaitement intégrée dans l'archi tecture générale du dialogue, la praxis contribue aussi à lier les deux grands thèmes de la conversation, l'amour et le mariage, qui, dans l'Antiquité, relè vent de deux problématiques philoso phiques différentes et dont l'unifica tion fait précisément aux yeux de M.
Foucault toute l'importance de notre texte $^{21}$. Pour les fondre, Plutarque doit assimiler mariage et amour conjugal ${ }^{22}$ et l'histoire d'amour romanesque qu'il a retenue l'y aide : Isménodore est tout à la fois amoureuse et désireuse de concrétiser sa passion par une union légitime 23 . Ce mariage, "le moins bon des mariages possibles 24 ", celui qui, précisément, est impensable dans le genre dominé par l'amour qu'est le roman ${ }^{25}$, par l'accumulation même des paradoxes $^{26}$, constitue le meilleur ali ment pour la réflexion philosophique, fille, comme on sait, de l'étonnement. Comme le soulignent sa concurrence
18

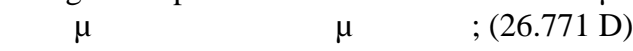
20

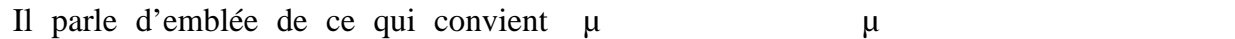

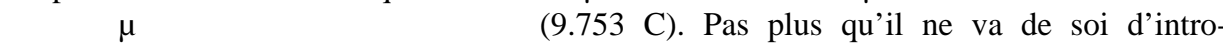
duire eros dans le mariage, il n'est courant d'assimiler amour des femmes et amour conjugal, comme en témoignent les débats des Amours transmis dans le corpus de Lucien ou ceux du livre II du roman d'Achille Tatius.

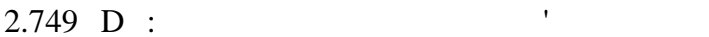

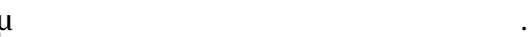
Fouc aul t , 1984, 227.

Les rapports de L'Érotikos avec le roman sont moins simples qu'il n'y paraît à première vue; le texte se situe à peu près sûrement à la fin de la vie de Plutarque, c'est-à-dire dans la période qui correspond en gros à la rédaction du roman de Chariton et où l'on peut penser que se développe un goût pour les histoires d'amour sensible en particulier dans les Vies de Démétrios et d'Antoine, elles aussi tardives. Mais on ne peut guère aller audelà de cette "ambiance" d'époque; dans le roman, l'amour est réservé au couple juvé nile et la veuve, même quand, cas rarissime, elle est sympathique, comme la Mélitè d'Achille Tatius, ne réussit jamais à désunir le couple juvénile. 
avec Pisias ${ }^{27}$, les reproches railleurs de Protogène, qui lui souffle la conduite à tenir ${ }^{28}$, et l'enlèvement drolatique inventé par Plutarque ${ }^{29}$, Isménodore tient le rôle d'un éraste : elle est, comme un éraste, plus âgée que son aimé; elle est, comme un éraste, habitée et inspirée par le dieu ; or, une honnête femme n'est pas censée avoir de rapport avec eros ${ }^{30}$. En elle s'in carnent ainsi les liens de l'amour et du mariage et poser son cas, c'est comprend re que la réflexion sur la valeur amoureu se du mariage passe par une réhabilitation de l'amour des femmes, c'est-à-dire par l'affirmation qu'elles peuvent être à la fois objets et sujets d'amour.

Cependant si Isménodore incarne avec une relative facilité ${ }^{31}$ cette unité, celle-ci a plus de mal à s'imposer dans la conversation. Sans doute, YÉrotikos, par opposition aux multiples questions que suscite la périégèse delphique du De Pythiae oraculis ou aux sujets divers abordés dans le De genio Socratis, ne traite-t-il que d'un seul sujet, clairement spécifié aux deux bouts du récit : on a débattu péri Erôtos ${ }^{32}$ et, après une sorte de prélude 'joué' par Daphnée et Protogène, la discussion est dominée par Plutarque -indéniable facteur d'u nité, mais qui reste très formel, alors que le contenu même de la discussion, avec les différentes facettes d'Éros, paraît se développer par 'tranches' suc cessives $^{33}$, dont l'articulation logique laisse souvent perplexe.

Leurs présentations successives au chapitre 2 font le portrait de personnes éminemment respectables, mais, en dehors de cette équivalence morale, qui permet au problème amoureux d'être posé dans toute sa pureté (l'un et l'autre sont animés des meilleures intentions possibles : reste à savoir ce qui est le meilleur pour Bacchon), Plutarque fait

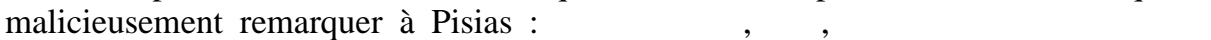

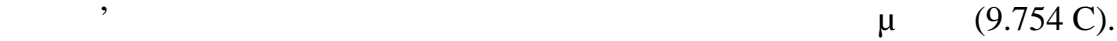

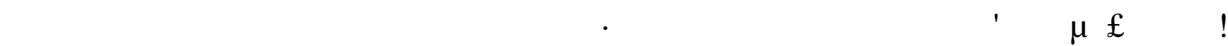

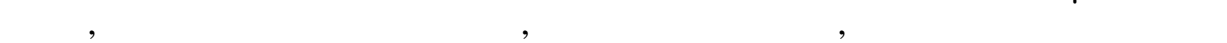
$\dot{\alpha} v \tau \varepsilon \rho \alpha \sigma \tau \dot{\alpha} ;(8.753 \mathrm{~A}-\mathrm{B})$

On songe à la fois aux enlèvements de comédie (mais c'est le jeune homme qui sauve la jeune fille du leno) et aux enlèvements pédérastiques de Crète (cf. Dov v r, 1982, 230).

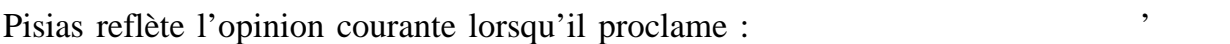

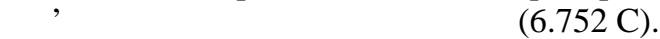

Relative, puisque justement son aventure est exceptionnelle, voire scandaleuse; d'où l'a nalyse de Goldhil 1, 1995, 158-161, qui insiste sur la tension entre l'histoire et le dialo gue et les ambiguïtés du désir féminin.

1.748 E et $26.771 \mathrm{D}$; curieusement $\mathbf{F l a c e l i e ̀ r e , ~ 1 9 8 0 , ~ i m p r i m e ~ d a n s ~ l e ~ p r e m i e r ~ c a s ~}$

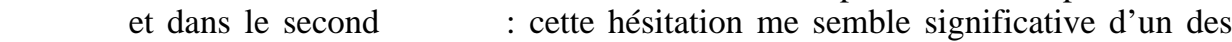
enjeux majeurs du texte ; il s'agit pour Plutarque de montrer derrière la manifestation psychologique et humaine la puissance du Dieu qui en fait toute la valeur. 


\section{Une discussion stratifiée}

Si l'on examine donc chacune de ces tranches, qui se distinguent par leurs inter locuteurs et leur tonalité propres autant que par la différence des sujets, on trouve d'abord un premier débat, aux chapitres 36, dominé par Protogène et Daphnée, qui se font les avocats de Pisias et Anthémion, c'est-à-dire les champions, le premier, de la pédérastie, et le second, de l'amour féminin identifié avec l'amour conjugal. La discussion se situe sur un plan général, sans référence au couple thespien ; mais, si le thème paraît $a$ priori relever de la synkrisis rhétorique, Plutarque le traite sur un mode plus philosophique, axant la con troverse non sur la valeur respective et la hiérarchie des deux amours, comme on l'attend d'une synkrisis, mais sur la natu re de l'amour, ou, plus précisément, sur la définition du concept : à quoi donner véri tablement le nom d' $\operatorname{eros}^{34}$ ? Et, du mo ment que les deux interlocuteurs s'accor dent pour reconnaître que l'amour est un, que recouvre cette unité? le seul amour philosophique ou tout amour sincère, indé pendamment du sexe de son objet ${ }^{35}$ ? Pour animé qu'il soit et jalonné de caricatures incisives $^{36}$ et de comparaisons piquantes ${ }^{37}$, le texte thématise donc d'entrée le problè me philosophique latent dans l'histoire d'Isménodore et de Bacchon : cette tonali té philosophique est appuyée dans le pre mier débat par la coloration stoïcisante des définitions de Protogène ${ }^{38}$ et les références platoniciennes de Daphnée ${ }^{39}$, tandis que la mise en question du mariage s'accuse en suite dans les commentaires contraires de Pisias, qui rejette avec force tout lien entre les femmes et $\operatorname{eros}^{40}$, et de Plutarque, qui refuse au contraire qu' on exclue eros du mariage ${ }^{41}$. La fin de ce premier mouve ment est bien marquée par la première intervention d'Anthémion, qui invite l'as semblée à "commencer à parler de notre sujet" (6.752 E).

Débute alors une seconde séquence (chs 7-9), toute différente de la premiè-
34

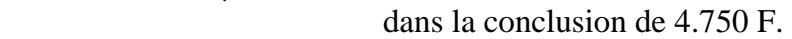

35

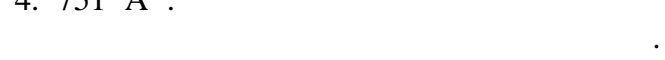

36

Voir l'opposition du rude amour philosophe et du voluptueux amour des femmes en 4.751 A-B et la peinture parallèle de l'Amour des garçons se glissant au gymnase et jouant au philosophe en 5.751 F-752 A.

37 Elles sont surtout l'apanage de Protogène qui veut réduire l'amour des femmes à un instinct naturel; voir 4. $750 \mathrm{C}$ et l'anecdote de Laïs en $750 \mathrm{D}-\mathrm{E}$.

38

39

40

41

VoirBABUT, 1969, 110-111.

5.751 E, où il se réfère successivement au Phèdre (1.1) et aux Lois (1.5).

6. 752 C, cité supra n. 30.

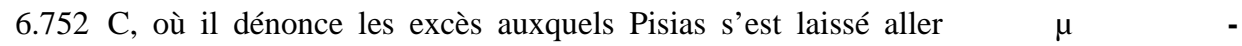

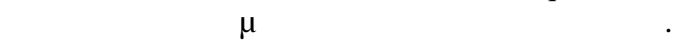


re, où, après que Pisias et Protogène ont exposé leurs objections aux prétentions d'Isménodore -rang social trop élevé et volonté de dominer pour le premier ; âge trop élevé et conduite indécente pour le second-, Plutarque prend longuement la parole comme "choreute de l'amour conjugal42", chargé par Daphnée de défendre à la fois l'amour et la riches $\mathrm{se}^{43}$. En fait, il insiste beaucoup plus sur le second point : axant sa réfutation sur le point de savoir quel genre de femme il faudrait épouser si l'on rejetait en Isménodore amour, richesse, jeunesse, beauté et noblesse, il effleure à peine le scandale d'une femme amoureuse en caricaturant à l'inverse les femmes sa ges, transformées en viragos de comé $\mathrm{die}^{44}$, et s'étend longuement sur le pro blème social, lié à la question de l'auto rité dans le couple. On est passé d'une discussion théorique sur l'être de l'a mour à des considérations pratiques sur la conduite dans le mariage, qui relèvent plutôt de la parénétique, comme en té moignent les nombreux points de con tact avec les Préceptes conjugaux ${ }^{45}$, et prennent même des accents de diatribe, multipliant interrogations rhétoriques et anecdotes pour réduire par l'absurde les allégations adverses. Mêlant ainsi apolo gie et polémique, le texte ne laisse pas néanmoins de faire apparaître une pre mière esquisse de l'idéal conjugal de Plutarque, qui refuse de considérer les avantages matériels comme un critère déterminant ${ }^{46}$ et insiste sur sa dimension morale. Seules ses vertus permettent à l'époux de diriger le couple et vouloir rabaisser sa femme pour la dominer plus sûrement est indigne : une certaine réci procité se fait ainsi jour et le mariage apparaît comme le moyen de s'élever ensemble. S'appuyant sur la situation paradoxale qu'il s'est donnée, Plutarque peut même, non sans quelque provoca tion, suggérer, au terme d'une longue litanie des autorités successives aux-

42 Ce qui est dans la continuité de sa rapide intervention de la séquence précédente.

43

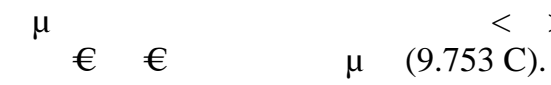

44

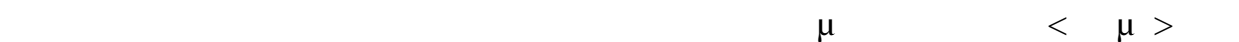

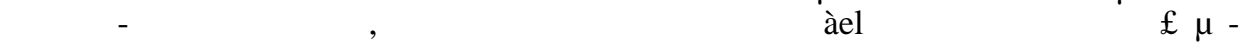

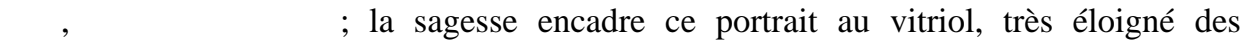
réflexions de Praec. conj. 142 B sur le même sujet : Plutarque se laisse visiblement emporter par la polémique.

45 Outre le passage cité à la note précédente -et où il y a un décalage-, comparer par exem ple 7.752 E et Praec. conj. 140 F; 9.754 A et 139 B; 9.754 D et 157 D, qui sont écrits dans un esprit voisin.

46

Pas plus dans un sens que dans l'autre, comme le montre la belle formule morale de

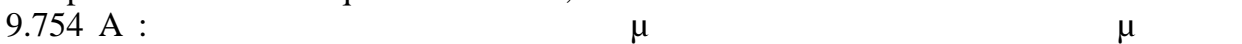

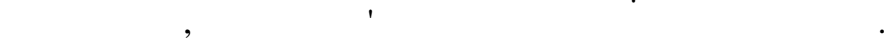


quelles un homme est soumis au cours de sa vie ${ }^{47}$, qu'il n'y aurait "rien de ter rible" à voir Isménodore, supérieure en âge et en sagesse ${ }^{48}$, "piloter la vie de son jeune mari49" : il propose ainsi qu'elle remplisse, dans le cadre du mariage, le rôle formateur de l'éraste.

Elle prend en effet la direction des opérations en enlevant Bacchon et, les acteurs du drame partis pour Thespies, la discussion revient à un plan général, mais sur un ton nouveau. La question posée par Pemptidès ressemble fort en effet à un thème philosophique qui serait proposé au professeur Plutarque :

Je suis resté silencieux jusqu'à présent, parce que la discussion me paraissait porter sur des ques tions personnelles plutôt que sur des idées générales, mais mainte nant que Pisias est éloigné, je vous entendrais volontiers ${ }^{50}$ ex pliquer quelle a été la pensée de ceux qui, les premiers, ont fait d'Éros un dieu. (12.755 F-756 A).
C'est ce thème que Plutarque déve loppe dans un long éloge du Dieu qui occupe les chapitres 13 à 18 : il établit d'abord la divinité, ancienne et vénéra ble, d'Éros, chargé de patronner l'amour et d'inspirer les amants (13-16.759 D); puis il la célèbre sous les deux rubriques de la puissance (16.759 D-17.762 A) et de l'utilité (17.762 B-18.763 B); enfin il conclut en la réaffirmant, s'appuyant pour ce faire sur la célébration unanime de toute la tradition, poètes, législateurs et philosophes (18.763 B-F). Ce premier ensemble se caractérise par un mélange constant de didactisme et d'éloge ; l'ex posé théologique initial (13-16) dénon ce le danger des pratiques allégoriques et affirme la sollicitude divine; la partie centrale justifie par une introduction théorique la double division de l'expo sé, entre puissance et bienfaits du dieu d'abord $^{51}$, puis, pour la première partie, en puissance supérieure à Aphrodite et puissance supérieure à Arès ${ }^{52}$; enfin la

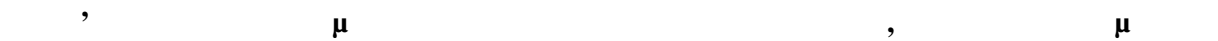

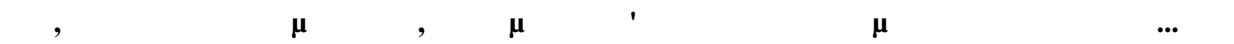

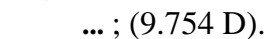

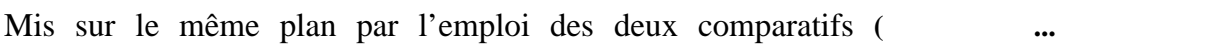
$\dot{\alpha} \lambda \lambda \mathbf{o v}$ ), l'âge devient ainsi synonyme de sagesse et donc, à la limite, un avantage plus qu'un inconvénient.

Plutarque remplace in extremis le $\dot{\alpha} \boldsymbol{\rho} \boldsymbol{\varepsilon} \mathbf{\imath}$ de la protase -employé aussi par Pisias- par

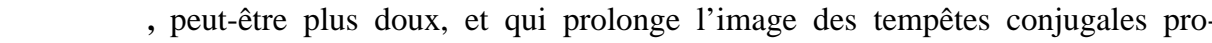
voquées par la jeunesse des époux.

Le verbe peut évoquer les auditeurs de cours de philosophie interrogeant le conférencier. Cette articulation sera en effet respectée (762 B).

Cette division s'appuie sur l'idée que la puissance de tous les dieux pourrait se ramener à celles d'Aphrodite et d'Arès, la première représentant la tendance de l'âme à s'appro prier le bien, la seconde, celle à lutter contre le mal, selon une division qui remonterait 
conclusion est fondée sur la theologia tripertita ${ }^{53}$.

Ce développement ayant donné lieu à deux allusions à la vision métaphysique de l'amour développée par Platon ${ }^{54}$, c'est au tour de Soclaros d'intervenir et de demander au professeur Plutarque de "révéler et dévoiler l'allusion qu'(il) a faite à l'accord 55 du mythe égyptien et de la doctrine platonicienne" : s'ouvre alors une séquence (19-20.766 B) à tona lité mystique, ou même mystérique, qui développe, en s'appuyant sur l'opposi tion du soleil et d'Éros et en multipliant les images (nuages; opposition du rêve et de la veille; arc-en-ciel), l'itinéraire de l'âme amoureuse, guidée par Éros mystagogue jusqu'à la beauté divine et intel ligible $^{56}$. On atteint là le telos de la phi losophie, cette partie que Platon et Aris tote auraient appelée "époptique ${ }^{57 "}$ et le lecteur aimerait, pour la satisfaction de son esprit logique, avoir atteint aussi le terme du discours. Mais Plutarque une nouvelle fois se dérobe et coupe : "Mais ces considérations dépassent le sujet de notre entretien" (20.766 B-C) et il re vient à la vie terrestre et au style anec dotique pour montrer comment le toutpuissant Éros se venge de ceux qui refu sent de l'accueillir à travers l'exemple d'amants méprisés, dont on ne sait s'ils se limitaient à l'histoire, inachevée dans notre texte, de Gorgo et d'Asandros.

En tout cas, l'intervention perdue de Zeuxippe, qui apparaît ailleurs comme un bon connaisseur, voire un sympathisant de l'épicurisme ${ }^{58}$, ramène sur le tapis les questions laissées en suspens après l'en lèvement de Bacchon et singulièrement

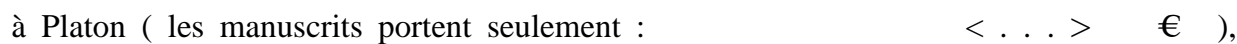
ce qui n'est pas la moindre énigme : le Phèdre, proposé par Fla a el ièr e, 1980, 142, note ad loc., ne convient pas, les chevaux de l'attelage ailé ne sont pas tous les deux bons; quant à la tripartition de République IV $439 \mathrm{E}$, suggérée par Barigazzi, 1986, elle explique bien l'assimilation du thymoeides à Arès, mais laisse pendant le cas d'Aphrodite, qui n'est à l'é vidence ni la raison ni le désir. En outre, ce qui épaissit encore plus le mystère, il faut ajou ter que cette théorie est complètement décalée par rapport à la suite du texte où, banalement, Aphrodite renvoie à l'amour physique et Arès au courage guerrier.

Consulter sur ce point l'article de G. Lieberg, A. N. R. W. I. 4, 1973, 63-115; on peut ajouter que l'exposé des dissensions des trois traditions fait la part belle à la tradition philosophique (18.763 C-D).

$17.762 \mathrm{~A}$ (à propos d'Éros, seul vainqueur d'Hadès) et $18.763 \mathrm{~F}$ (dans l'image finale du cortège d'Éros salué par la tradition).

Ce thème de la "concorde" ou de l'accord est aussi un thème exégétique familier aux philosophes platoniciens.

Sur ces images, voir Frazier, 1999.

57

58

De Iside $77.382 \mathrm{D}$.

Voir Pu ec h, 1992, sv. "Zeuxippos”, p. 4891. 


\section{L'Érotikos : un éloge du Dieu Eros? Une relecture du dialogue de Plutarque 73}

celle de l'amour des femmes. Peuventelles séduire ? Tel est le premier thème qui devait être traité ; dans son état actuel, notre texte reprend au chapitre 21 , à la fin de la démonstration, avec un ultime argu ment ${ }^{59}$, qui, entre polémique et apologé tique, multiplie les parataxes pour mon trer l'absurdité qu'il y a, quelque théorie que l'on adopte, épicurienne, platonicien ne ou stoïcienne ${ }^{60}$, à refuser aux femmes le pouvoir de susciter l'amour.

Après quoi il rappelle l'assimilation de l'amour à une passion débridée à laquelle Zeuxippe, dans l'esprit de Pemptidès au chapitre 12, mais aussi de Protogène au chapitre 4, aurait procédé61 et l'attribue aussitôt à des hommes dyskoloi et anerastoi qui, dans le mariage, ne verraient que l'argent ou le moyen de s'assurer une des cendance et ne se soucieraient "ni d'aimer ni d'être aimés ${ }^{62}$ " : en opposition, il déve loppe sa propre conception du mariage, qui est fusion et fidélité, illustrée à nou veau par des exemples qui culminent avec l'histoire de Camma (22).
Ce cadre moral fermement dessiné, il peut revenir aux points litigieux en repre nant à nouveau des objections de ses adversaires et traiter de la place de l'a mour physique et de l'aptitude des fem mes à la philia ${ }^{63}$ : une première célébra tion enthousiaste de la beauté du mariage s'exalte jusqu'à évoquer une sorte d'hié rogamie du dieu et de la matière et est coupée d'un "Mais pour ne pas avoir l'air de m'écarter trop loin ou de bavarder tout à fait à tort et à travers..." ; on revient alors à la stabilité et fidélité des couples d'amoureux véritables ${ }^{64}$ et à un nouveau récit, l'histoire d'Empona (25).

Cette analyse, rapide, destinée d'a bord à fixer le mouvement du texte et ses grandes parties, fait bien apparaître, en dépit du facteur d'unification qu'est la prééminence de Plutarque, qui mono polise à peu près constamment la parole à partir du chapitre 9 , le mélange perma nent de théories discursives et d'illustra tions narratives et la grande diversité des tons, tantôt rhétorique et tantôt plus phi-
59

60

Seules les deux premières sont nommément citées (766 E), mais la troisième est aussi

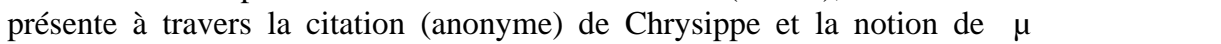

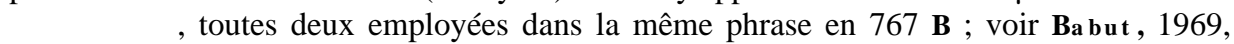
111-112 et infra p. 89.

61
$767 \mathrm{D}$, qui répond à $6.752 \mathrm{C}$ (appliqué aux femmes).

23.768 E : "Qui supporterait ceux qui outragent Aphrodite...?"; 769 B : "Mais (dira-ton) bien des passions criminelles et furieuses sont nées de l'amour des femmes"; 24.769 E : "Quant à cette douleur mordante du début, très cher Zeuxippe, ne la crains pas...".

On touche là l'infériorité majeure de la pédérastie : les "amants véritables" le restent toute leur vie, mais de tels amants sont rares. 
losophique, tantôt didactique et tantôt encomiastique, allant jusqu'au mysticis me, la multiplicité aussi des points de vue adoptés pour considérer l'amour : ainsi, au dieu traditionnel des chapitres 13-18 succède la figure de l'Éros plato nicien sans que l'articulation des deux soit très claire —on pourrait suggérer une sorte d'effet de 'fondu', qui repren drait sur un autre plan, plus élevé, l'accord des poètes, des législateurs et des philoso phes sur un Dieu en le faisant apparaître dans toute sa puissance ; mais si l'articu lation des deux parties du discours central peuvent susciter des interrogations, plus importante encore pour le sens général du dialogue est l'interprétation du lien qui unit l'ensemble de ce développement cen tral à l'apologie du mariage, sujet des deux discussions qui l'entourent.

L'articulation de l'éloge central d'Eros et de l'apologie 'périphérique' du mariage.

11 est d'autant plus délicat de voir comment les deux thèmes pouvaient s'ar ticuler dans la pensée que nous ne voyons même plus comment matériellement on passait de l'un à l'autre dans le texte. À quelle occasion Zeuxippe intervenait-il ? On peut supposer que les anecdotes fina les du grand discours, l'histoire de Gorgo, facilitaient le retour au thème du mariage, mais, quelle qu'ait été la technique adop tée par Plutarque, il n'en reste pas moins que l'état actuel du texte accentue encore la disparate entre les deux développe ments, ce qui a amené les critiques à iso ler totalement l'éloge central, replacé dans la tradition de l'eros philosophique, de l'apologie périphérique du mariage, qui refléterait davantage l'évolution des mœurs, puis, cette distinction opérée, à apprécier, en fonction de leurs préoccu pations personnelles, l'importance de chaque développement. L'analyse de R. Flacelière est très représentative de cette manière de poser le problème ${ }^{65}$ :

Les nombres de pages consa crées à chacun de ces deux sujets s'équilibrent sensiblement ${ }^{66}$, en sorte qu'il paraît difficile de savoir si Plutarque attachait plus d'importan ce à l'un ou à l'autre, et si l'on doit considérer la partie centrale comme une digression, ou, au contraire comme l'essentiel de l'ouvrage.

Pas un instant il n'est envisagé que les deux aspects soient également importants ; en conséquence de quoi, R. Flacelière affir me avec force la primauté de l'apologie de l'amour conjugal au motif qu'il est le point sur lequel Plutarque achève le dialogue. Plutarque userait ici de la même technique que dans le De Pythiae oraculis, qui doit être à peu près contemporain :

De même que, dans le dialogue Sur les oracles de la Pythie, toutes les idées auxquelles Plutarque tient
65

66

Fl a c el ièr e e, 1980, 20.

Déjà peu probant en soi, cet argument l'est encore moins si l'on songe que notre texte souffre d'une lacune, que les spécialistes estiment, les uns à un simple feuillet, mais les autres à un cahier entier, ce qui modifierait considérablement les équilibres. 


\section{L'Érotikos : un éloge du Dieu Éros? Une relecture du dialogue de Plutarque 75}

visiblement le plus sont exposées par Théon à la fin de l'ouvrage, nous pouvons être sûrs qu'il a écrit l'Éroticos surtout pour réfuter la thèse des partisans de la pédéras tie, pour montrer la beauté et la grandeur de l'amour conjugal.

Or il n'est pas sûr du tout que les deux buts ainsi assignés à Plutarque se recouvrent. De fait, c'est surtout la re vendication d'exclusivité de la pédéras tie qui est réfutée afin que sa place légi time soit faite aussi à l'amour conju gal ${ }^{67}$; mais les deux ne semblent pas a priori s'exclure et le rapport ne tend à se renverser que tout à la fin, quand est envisagée la question de la fidélité ${ }^{z 0}$ : celle des pédérastes n'a pas grande répu tation, mais, après avoir rapporté les railleries que suscite leur inconstance, Plutarque nuance aussitôt : "toutefois il n'est pas juste de faire ce reproche aux

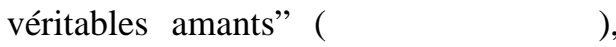
citant le beau mot d'Euripide, fidèle à
Agathon, avant de conclure que "si l'on ne peut dénombrer que quelques couples pédérastiques, il y a des myriades de couples avec des femmes qui ont conser vé jusqu'au bout, fidèlement et ardem ment, la cohésion d'une fidélité abso lue" $(770 \mathrm{C})$. La constatation est pure ment 'historique' : tels sont les faits, mais les couples fidèles masculins ne sont ni moins possibles en théorie ni moins louables en pratique. Néanmoins, il est indéniable que réhabiliter avec éclat les aphrodisia, lorsqu'ils vont de pair avec eros, et faire de l'union char nelle un ciment de l'entente sentimenta le, met nécessairement en situation d'in fériorité l'amour pédérastique à qui ces pratiques sont interdites, et l'on com prend que, dans le traité antistoïcien Sur les notions communes, s'il n'impute aux Stoïciens et à leur amour philosophique aucune immoralité, il reproche au se cond de ne pas être de l'eros et aux pre miers de mésuser des mots ${ }^{69}$.

Même Daphnée, qui est plus virulent que Plutarque, rejette sur l'intransigeance de Protogène la condamnation sans appel de la pédérastie qu'il prononce $(5.751 \mathrm{~F}$ : et òè

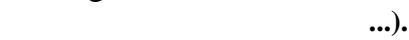

24.766 B : "Ne sais-tu pas que l'amour des garçons provoque beaucoup de blâmes et de railleries pour cause d'inconstance?"

"Eh ! Que sommes-nous donc en train de faire d'autre, mon excellent ami, sinon de convaincre leur école de disloquer les notions communes que nous détenons et de leur faire violence en s'appuyant tant sur des faits qui ne sont pas plausibles que sur des expressions qui sont contraires au langage usuel ? Car nul ne les empêche d'appeler 'chasse' ou 'liaison d'une amitié' (фıдолоін $\alpha$ ) l'attachement des Sages aux jeunes gens, s'il est vrai qu'il ne s'y mêle pas de passion ; mais le nom d'amour (cpoos) <devait> être réservé à ce que tous et toutes conçoivent sous ce nom et désignent ainsi : 'ctous furent pris du désir> d'être au lit, <auprès d'elle>, <et, jamais encore pareil> désir <d'une déesse> ni d'une femme / n'a à tel point inondé et dompté <mon cœur> dans ma poitrine'" (1073 C, trad. D. Ba but ). 
La chose n'est pas si claire dans l'Érotikos, mais si l'on ne peut parler sans forcer le texte de condamnation de la pédérastie, on ne peut non plus nier le vibrant éloge du mariage qu'il contient. Cependant à trop l'accentuer, on crée un nouveau déséquilibre : le discours cen tral semble en porte-à-faux, simple di gression, suggère R. Flacelière dans sa notice, pure concession à la tradition, estime L. Goessler ${ }^{70}$, qui ne voient l'un comme l'autre dans ce texte qu'une cé lébration de l'amour pédérastique. Or, à nouveau, une telle analyse simplifie à l'excès les choses et il est très remarqua ble au contraire que, chaque fois que l'on est dans un contexte fortement pla tonicien, qu'il soit question des maniai (16.759 A), des vestiges de beauté que l'amant aperçoit dans l'aimé (20.765 F) ou au contraire de ceux qui sont trop attachés aux corps $(20.766 \mathrm{~B})^{71}$, Plutar que prend toujours grand soin d'associer dans ses définitions filles et garçons comme objets d'amour ${ }^{72}$ et qu'il puise ses exemples dans tous les cas de figures
: amants rendus héroïques sur le champ de bataille par la présence de leur aimé ou tyrannoctones, mais aussi compa gnons d'Alexandre épris de musicien nes, Alceste éprise d'Admète ou Sapho chantant son aimée.

La différence entre les deux dévelop pements ne tient donc pas au sexe de l'objet aimé, mais bien plutôt au point de vue adopté. Le discours central est consacré à un exposé théologico-philosophique, qui insiste sur la nature et les effets spirituels de l'amour avec des accents religieux et mystiques tandis que les développements sur l'amour conju gal réintroduisent la dimension charnel le et insistent sur la fusion et la fidélité des conjoints : on a entre les deux toute la distance qui peut séparer l'abstrait du concret, le théorique du pratique, le métaphysique de l'éthique, ce qui ne signifie pas qu'ils soient étrangers l'un à l'autre, ni même étanches. Le discours central se nourrit d'exemples concrets ${ }^{73}$ et la célébration finale du mariage

Goessl e r, 1962, 42 : "Der Grund dieses Widerspruches liegt -so darf wohl mit Bestimmtheit gesagt werden- in einer liebenswürdigen Schwàche des Schriftstellers und des Menschen Plutarch. Er steht im Amatorius durchaus in der Tradition $\pi \varepsilon \rho i$ E $\rho \omega \tau$, und in dieser halten, nicht bloB für ihn, Platon und somit auch der $\pi \alpha 1 \delta$ เкó E $\rho \omega$ des Symposion und des Phaidros maBgebliche Geltung"; on trouve encore chez Canta rell a, 1991, 112, l'idée, qui me semble peu défendable, que "pour lui, l'amour le plus noble était l'amour entre hommes."

Les deux premiers thèmes viennent du Phèdre et le dernier est une adaptation du Phédon.

La seule exception que l'on trouve n'est justement pas dans un contexte platonicien, mais dans une argumentation ; Plutarque ayant expliqué que toutes les nécessités de la vie (nais sance, maladie, mort) avaient un dieu pour les patronner, affirme que rien n'est plus beau et

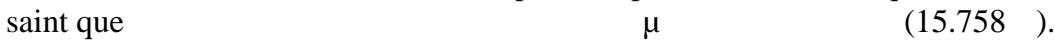

Sur leur fonction, Fr a z ie r, 2005a. 


\section{L'Érotikos : un éloge du Dieu Èros? Une relecture du dialogue de Plutarque 77}

s'exalte jusqu'à évoquer l'hiérogamie du dieu et de la matière ou, pour le dire dans les termes de R. Flacelière, jusqu'à "s'é garer un instant, en $770 \mathrm{~B}$, dans des consi dérations de haute métaphysique sur le rôle que joue Èros dans la vie cosmique", et l'éditeur de s'interroger: "Ces 'égare ments' sont-ils toujours involontaires ? Plutarque n'entendait-il pas imiter la fantaisie des dialogues platoniciens par cette libre allure d'un entretien semé de digressions ?74". Mais pas plus que les 'détours' platoniciens ne sont insignifiants —Victor Goldschmidt a pu au contraire les qualifier de "détours essentiels"-, ils ne sont chez Plutarque ni une simple imi tation, l'élaboration d'un "à la manière de Platon", ni le fruit d'une composition non chalante : chez le maître athénien comme chez son lointain disciple chéronéen, ils sont l'expression d'une pensée vivante, d'une réflexion toujours en alerte et prête à se saisir des sujets qui se présentent. On retrouve cette même tendance dans les Propos de table, où Plutarque et ses amis peuvent prendre pour prétexte à discus sion un poisson que l'on sert à table, les arbres qui ombragent un jardin ou un bruit qui résonne du dehors; on la voit à l'œu vre aussi dans les Vies, où Plutarque s'attarde à expliquer la résurgence des sources ou à réunir, au fil de la plume et de ses souvenirs, tout ce qu'il peut savoir d'Aspasie. Plus que d'une imitation super ficielle, il s'agit d'une manière de réfléchir qui lui est propre et qui, dans le dialogue, ne l'éloigne pas de son sujet, mais lui per met d'en explorer toutes les implications.

Plus précisément, sa réflexion est animée sans cesse d'un double mouve ment, qu'a mis en évidence D. Babut ${ }^{75}$ : d'un côté, Plutarque a tendance à 's'é chapper' vers des considérations méta physiques et à dessiner un idéal, de l'au tre il revient sans cesse aux réalisations pratiques, à la vie courante dans laquel le il s'agit de 'bien vivre' et de réaliser, autant que faire se peut, le te los défini par la philosophie . La chose est cons tante chez lui : elle est en accord avec l'esprit même de la philosophie antique et, en premier lieu, de Platon, qui n'a jamais envisagé que le philosophe pût ne pas redescendre dans la caverne; elle convient tout particulièrement à l'a mour, qui est une réalité essentielle de la vie humaine, à l'origine de cette vie et sans cesse présente tout au long de son déroulement; là encore il peut s'autori ser de Platon et du discours d'Alcibiade, que l'on peut lire comme une concréti sation du discours de Diotime, permet tant à l'amour philosophe de s'incarner sur terre dans la personne de Socrate.
75

76

Cf. Ba but , 1994, 576 : "C'est que conformément aux tendances de son époque, la philoso phie est avant tout pour lui un 'art de bien vivre', de sorte qu'il voit surtout dans ses maît res ceux dont l'enseignement nous aide à mieux vivre plutôt que des 'maîtres de vérité'." 
Au lieu de considérer le discours cen tral comme secondaire, parce qu'il n'est pas le dernier mot de Plutarque, on peut ainsi noter tout au contraire qu'il a été placé au cœur même du dialogue, dont il établit les fondements, et relever à quel point il est enserré dans l'évocation de l'amour vécu : non seulement, il est en touré par deux discussions sur l'amour conjugal (3-9 et 21-25), mais lui-même, après l'exposé platonicien des chapitres 19-20, revient à des histoires d'amour, donc à des amours vécues, avec l'histoire de Gorgo, c'est-à-dire qu'il renoue avec un style qui était celui de la première intervention de Plutarque à propos de l'a mour conjugal, au chapitre 9 , où il évo quait l'histoire de Ninos et de Sémiramis, mais aussi celui de la première partie du discours. Ces remarques, si elles ne doi vent pas effacer la diversité des tons et des points de vue qui se succèdent au fil du texte, invitent à le reprendre dans son ensemble en étant attentif, non plus aux éléments de variété, mais d'unité, à rele ver les thèmes, qui, par leur récurrence, construisent une certaine image d'Éros propre à l'Érotikos : l'image d'un dieu, dont Plutarque célèbre la puissance et les bienfaits en nourrissant son éloge de tous les éléments de la tradition.

Un éloge de la puissance et des bien faits du Dieu

La nature d'Éros : theos et pathos

Un des points les plus discutés de $Y E$ rotikos, sans qu'on en mesure toujours toute l'importance, est la nature d'Éros : c'est le sujet de la première discussion qui oppose Protogène et Daphnée, une discus sion dont la chaleur même est révélatrice et à laquelle Pemptidès fait allusion lors qu'il intervient :

Pemptidès sourit : "Il est vrai, dit-il, qu'il existe une maladie du corps que l'on appelle le mal sacré; il n'y a donc rien d'étonnant que la passion de l'âme la plus furieuse et la plus forte ( $\psi v \times \dot{n}$

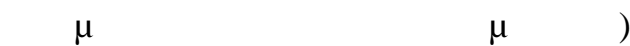
soit dénommée par quelques-uns sacrée

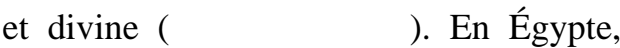
un jour, j'ai vu deux voisins se disputer au sujet d'un serpent qui s'était avancé sur le chemin ; tous les deux l'appelaient un bon génie, mais chacun le revendi quait pour sien; quand je vous ai vus pareillement tout à l'heure tirer Eros, les uns vers les lieux de réunion des hom mes, les autres vers le gynécée, en le considérant tous comme un bien mer

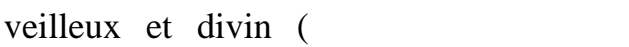
$\alpha \gamma \alpha \theta$ òv), je n'ai pas été surpris que cette passion $(\pi \dot{\alpha} \theta \mathrm{o})$ jouisse d'une si grande puissance et de si grands honneurs, quand ceux qui devraient la chasser de toutes parts et tâcher de l'amoindrir la font croître et la célèbrent ( $\alpha \dot{v} \xi \alpha v o \dot{~ \varepsilon v o v}$

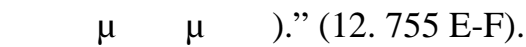

Ainsi est soulevée la question de la divinisation de l'amour, qui marque le début de l'intervention centrale de $\mathrm{Plu}$ tarque. On se trouve donc devant l'alter native theos ou pathos: Plutarque ne re prend pas l'analyse du Banquet et la seu le occurrence de daimôn qu'on trouve dans Y Érotikos est pour ce serpent égyp 
tien évoqué par Pemptidès ${ }^{77}$. Cette ou un démon ou un état de l'âme ${ }^{80}$ et divergence est constamment relevée, s'efforce de concilier ces trois interpréta sans commentaire parfois, le plus sou tions. Or il faut noter que, pour examiner vent avec quelque réprobation, comme ces trois possibilités, il sépare son expo si Platon n'avait parlé d'Éros que dans le sé en deux parties, distinguant $\Gamma$ amourBanquet, comme si, dans le Phèdre, il état de l'âme et Гamour-être divin, lequel n'était pas un Dieu, comme si Plutarque se subdivise à nouveau en daimôn et devait se rallier sans discussion à ce qui theos selon le niveau de réalité auquel on semble être la vulgate scolaire d'après se situe : on retrouve, associés dans une les manuels d'Alcinoos ou d'Apulée, vision stratifiée de la réalité, les deux comme si la tradition platonicienne n'é aspects disjoints dans l'alternative de tait pas assez complexe pour que, plus Plutarque. Cette subdivision, qui n'est tard, Plotin se demande, dans son traité donc pas philosophiquement infondée, 50 (Ennéades III. 5), si Éros est un Dieu est par ailleurs fidèle aux emplois tradi-

77 La vénération du serpent sous le nom $\dot{\alpha}^{\prime} \alpha \gamma \alpha \theta^{3} \dot{\mathrm{o}} \delta \dot{\alpha} \mathrm{i} \omega v$ ést une réalité de la religion égyptienne dont je dois la connaissance à mon collègue Christophe Chandezon. Pour être complet, il faut signaler un autre emploi de $\delta \alpha i \omega v$ au ch. 25 , mais au pluriel, asso cié à Geoi pour désigner l'ensemble du monde divin ulcéré par la cruauté de Vespasien.

78 Même Fl acel ière, 1980, qui défend la fidélité de Plutarque à Platon, écrit : "Il est juste cependant de faire à la thèse de Hirzel une importante concession. On sait assez que dans le Banquet de Platon, Diotime, dont Socrate prétend rapporter les paroles, présente l'Amour comme un être de nature intermédiaire, comme un Génie ( $\delta \alpha i \omega v)$. Or, dans l'Éroticos, Éros apparaît toujours comme un dieu, et comme un dieu puissant; sa nature divine n'est jamais contestée par l'auteur et les doutes de Pemptidès à cet égard dont énergiquement écartés."

79 Alcinoos, Didask. XXIII. 183.74 sq : “Aussi faut-il représenter l'amour comme un démon plutôt que comme un dieu, puisqu'il n'entre jamais dans un corps terrestre, mais qu'il transmet aux hommes ce qui vient des dieux et réciproquement"; de même Apulée, lorsqu'il évoque les démons qui n'ont jamais été incarnés, donne pour exem ples Somnus et Amor (De Plat, et ejus dogm. 1.16.154-155); mais, dans son développe ment sur l'amour (ibid. 11.14.239 sq), les choses se compliquent et sont distinguées trois formes d'amour, l'amour ignoble qui est une maladie du corps, l'amour divin qui pénè tre dans les âmes humaines deorum munere beneficioque concessus, adspirante caelesti cupidine, et l'amour intermédiaire, résultant de la juxtaposition de l'amour divin et de l'amour terrestre.

Eros daimôn vient du Banquet, Eros theos et éros pathos viennent tous deux du Phèdre (respectivement 242 e et 252 b). Les commentateurs, anciens (ce qui n'est pas étonnant), comme modernes (ce qui l'est plus) ne cherchent pas sur ce sujet à replacer les concep tions de Platon dans le cadre spécifique de chaque dialogue : dans le Banquet, la réflexion sur le metaxu et la peinture de l'Amour comme philosophe excluent d'en faire un Dieu; un Dieu serait sophos et on ne pourrait lui identifier Socrate. 
tionnels du mot eros, qui peut désigner une passion furieuse comme l'amour philosophique le plus éthéré, c'est-à-dire, si l'on veut théoriser l'usage, présen ter l'amour comme un simple phénomè ne psychologique, passion funeste le plus souvent , ou comme un sentiment subli me qui n'est tel que parce qu'il a derriè re lui, ou au-dessus de lui, un Dieu. De cette tradition, le débat rhétorique des Amours conservé dans le corpus de Lu cien donne un bon témoignage :

L'Amour est un dieu double qui n'arrive pas toujours par la même voie et dont le souffle allu me dans nos âmes des feux bien différents. Mais l'un, selon moi, ne s'occupe que de puérilités; aucune raison ne peut guider ses sentiments; il se concentre avec une grande violence dans les âmes des insensés; c'est de lui que viennent surtout les désirs qui les entraînent vers les femmes. Cet amour accompagne toujours la fougue éphémère et précipite d'un élan invincible vers l'objet de la quête. Quant à l'autre Amour, ancêtre de l'âge d'Ogygès, vision vénérable et spectacle tout à fait sacré, propagateur des saines pas sions, de son souffle il pénètre en douceur dans les âmes de chacun; et quand ce dieu nous est propice, nous goûtons la volupté mêlée à la vertu. Car, en vérité, l'Amour, selon le mot du poète tragique, a deux souffles différents et sous un même nom, il engendre des pas sions opposées. De même la Pudeur est une double divinité, tout à la fois utile et pernicieuse : Pudeur à l'envi sert ou perd les hommes. Une seule lutte, n'en parlons point ! Ici-bas

Il en est deux; l'une sera louée de qui la comprendra,

Condamnons l'autre. Distance partage leur double cours.

Ainsi, l'on ne doit pas s'éton ner que l'on ait donné à la passion une dénomination qui ne convient qu' à la vertu et que l'on ait appe lé Amour la volupté déréglée et la sage bienveillance ${ }^{82}$.

Cette différence se fait jour aussi dans les commentaires totalement opposés que Plutarque fait des mêmes vers de Ménan dre : "L'amour est un kairos de l'âme..." dans les fragments du Péri Erôtos que nous a conservés Stobée et dans notre Érotikos. Le premier texte, dans l'esprit des petits traités parénétiques, approuve cette vision des choses :

C'est bien et justement dit. Il faut en effet qu'il y ait rencontre entre le patient et l'agent et une certaine disposition réciproque, car la force active est incapable de réaliser sa fin sans une disposition passive (qui l'accueille). Et il s'a-

\footnotetext{
81

D'après les fragments, ce devait être l'optique du $\pi \varepsilon \rho i$ êpurroç.

83 Ch. 37, trad. P. Ma r é c h a ux, éd. Arléa, 1993.

Voir Frazie r, 2003.
} 
git de bien viser le moment cri tique qui met juste à point en contact le principe naturellement actif avec l'élément passif prêt à le recevoir (frg. 134 Sandbach).

Le fragment s'interrompt là, mais on peut supposer que cette analyse amenait à déterminer dans quelles conditions on doit se trouver pour ne pas se laisser entraîner à une passion dont la cause est intérieure ; au contraire, dans VÉrotikos, Plutarque introduit la citation d'un "Non, je ne comprends ni ne conçois ce que Ménandre dit" et la rectifie aussitôt : “en réalité c'est le Dieu qui est cause de tout, s'emparant de l'un et laissant l'au tre" (18.763 B) ; la cause est extérieure et c'est comme enthousiasme que l'a mour prend toute sa valeur. L'introduc tion de la théorie platonicienne de la mania illustre bien l'effort de Plutarque pour magnifier l'amour et éviter sa ré duction à une passion funeste :

En ce qui concerne la mania, il en existe une forme qui vient $d u$ corps et se communique à l'âme et qui, produite par des humeurs ma lignes ou par les effluves d'un flui de pernicieux et subtil, est une ma ladie grave et dangereuse; mais il en est aussi une seconde à laquelle la divinité n'est pas étrangère et qui ne vient pas de nous, une inspira tion extérieure, qui dérange notre entendement et notre raison, dont l'origine et le mouvement viennent d'une puissance supérieure : c'est ce qui porte le nom générique d'enthousiasme' 84 .

Clair dans son énoncé, cet argument, qui constitue la réfutation la plus directe de l'attaque de Pemptidès, intervient cependant bien loin dans la réponse de Plutarque : c'est que la lumière s'est concentrée d'abord sur ce qui est le point essentiel, la divinité, dont l'impor tance primordiale ressort du mouvement même de la discussion.

Lorsqu'ils ont lancé le débat, Proto gène et Daphnée se sont accordés pour poser l'unité de l'Amour, en le situant déjà dans une ambiance plus ou moins imprégnée de sacré : Protogène a parlé des "noms les plus beaux et les plus

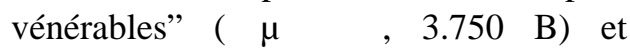
Daphnée a nié qu'il y eût "d'union plus

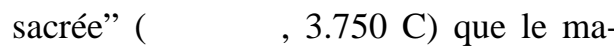
riage ; pour tous deux, comme pour Plotin, comme aussi, d'après la fin du texte des Amours, pour Callicratidas, il n'est d'Amour véritable que bon : la forme mauvaise, Vepithymia qui ne vise que le plaisir, ne mérite pas le nom d'amour ; c'est ce que conteste radicalement Pemptidès, qui réduit l'amour à un $\alpha v 1 \kappa \dot{\omega} \tau \alpha \tau o v \pi \dot{\alpha} \theta \mathrm{o}$. indûment divinisé. Plutarque est ainsi invité par lui à une

16.758 D-E ; tous les mots sont importants dans la seconde définition : $\dot{\varepsilon} \tau \dot{\varepsilon} \rho \alpha \delta^{\prime}$ où

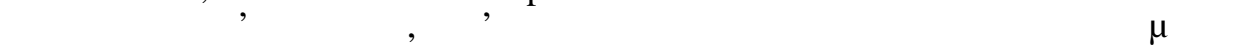

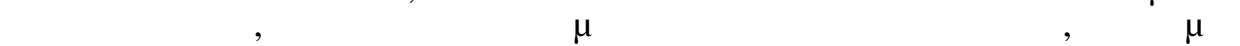

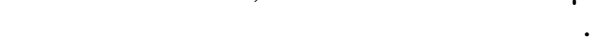


réflexion morale -comment a-t-on pu se laisser entraîner à cela ?-, mais ce n'est pas du tout dans cet esprit qu'il répond ; il ne parle pas d'abord de $p a$ thos., mais de theos, et voit dans la ques tion de son ami une remise en cause de tout l'édifice religieux. Comme dans le De Iside, il souligne le danger d'“ébran ler l'inébranlable ${ }^{85}$ ", récuse les interpré tations allégoriques ou evhéméristes qui "effacent" les divinités ${ }^{86}$, puis insiste sur la bonté divine et le patronage qu'appor tent les dieux à chacune de nos activités et de nos tendances, en interrogeant Pemptidès sur le rôle d'Arès ; celui-ci ${ }^{87}$ étant convenu qu'il ne se confondait pas avec le thymoeides en nous, mais le réglait, Plutarque peut lui présenter, en antithèse, la tendance patronnée par Éros

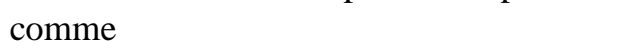

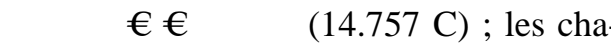
pitres 14 et 15 se développent ensuite en une succession ininterrompue de raison nements a fortiori, montrant que, si la chasse réelle est patronnée par une dées se, la chasse amoureuse doit l'être aussi; que, si la culture est protégée, le dévelop pement de l'être humain doit l'être aussi ; que, si un dieu s'attache à toutes les nécessités de la vie, l'amour est celle qui le mérite le plus ; le chapitre 16 poursuit dans le même style que si les trois autres formes de philiai ont un dieu, la philia érotique ne saurait en être privée avant d'introduire solennellement la théorie pla tonicienne de la mania : en d'autres ter mes, ce n'est qu'après avoir bien ancré l'i dée que l'amour se développe sous l'égi de d'un dieu, en n'utilisant que les aspects positifs de l'amour et son image de philia, que Plutarque aborde enfin son côté pas sionné, pour en faire, dans l'esprit même du Phèdre, le signe de l'intervention divi ne. La conclusion s'impose et réconcilie theos et pathos en montrant l'âme "com me portée sur un flot de passion en même temps que le dieu" (16.759 D).

Le thème revient encore pour cou ronner l'évocation des bienfaits qu'Éros apporte, non à l'aimé, car ils sont évi dents, mais à l'amant. L'exposé com mence sur un plan moral, en évoquant la générosité, la bonté qu'Éros développe chez l'amant, mais il s'attache bientôt au bouleversement que produit chez l'a mant la vision de l'aimé, illustré par la célèbre ode de Sapho que Plutarque invite Daphnée à réciter et qu'il com-
85

86 87

88

89

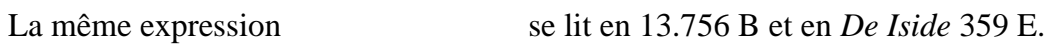

À nouveau le même verbe $\left(\delta 1 \alpha \gamma \rho \dot{\alpha} \varphi^{\wedge} 1 v\right)$ se lit en $13.757 \mathrm{C}$ et en De Iside $377 \mathrm{D}$.

Dont la réponse rend plus que douteuse l'étiquette d'épicurien qu'on veut généralement lui attribuer.

88 Ce passage (16.758 C-D) est intéressant car il emprunte déjà à l'enseignement platoni cien, si l'on en croit Diogène Laërce (3.81), mais en ajoutant à la liste 'normale' des trois philiai, la forme 'érotique' qui l'intéresse.

17.762 B - $18.762 \mathrm{E}$ 
mente en ces termes sans équivoque :

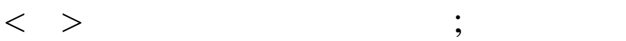

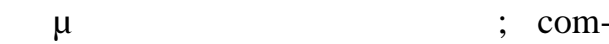
mentaire qui prélude d'abord à la cita tion de Ménandre, puis à la conclusion grandiose $^{90}$ qui célèbre l'accord excep tionnel de toute la tradition sur la divini té d'Éros : “il n'est qu'un dieu sur lequel leur accord est constant ${ }^{91}$ et unanime ment l'élite des poètes, des législateurs et des philosophes inscrivent Èros au nombre des dieux ${ }^{92}$, d'une seule voix le célébrant hautement, comme, selon Alcée, les Mytiléniens, quand ils choi sissaient Pittacos pour tyran ${ }^{93}$ ".

Cet ancrage dans la tradition se retrou ve encore dans la dernière partie consa crée à l'amour conjugal, où, pour confir mer l'importance de l'amour charnel dans l'union des époux, Plutarque s'appuie sur le nom d' 'Harmonie' donné par les Delphiens à Aphrodite, sur l'emploi par Ho mère du mot philotès et sur la loi de Solon imposant aux époux de s'unir au moins trois fois par mois afin de "renouveler leur mariage en effaçant les griefs accumulés au fil des jours au moyen de cette marque d'affection ${ }^{94}$ " (23.769 A-B). On touche là un des points importants de la représenta tion d'Éros.

\section{Le dieu de la tradition}

Conclue sur l'image triomphale d'É ros amené de l'Hélicon à l'Académie par "Hésiode, Platon et Solon" réunis" la réponse de Plutarque à Pemptidès avait commencé par une défense éner gique de la patrios pistis menacée par la suspicion $96 *$ et l'auteur semble attirer luimême l'attention du lecteur sur cet enca drement par une introduction rhétorique insistante : "Ce qu'il eût été plus à pro pos de dire au début, maintenant non plus 'puisque cela me vient à l'instant
90

Ǎ laquelle Fr a c el ière, 1980, 85, donne un sous-titre évocateur : "Apothéose de l'Amour'.

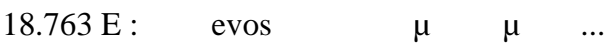

A้ opposer aux imputations de bâtardise de 4.750 F; 5.751 F, déjà niées en 13.756 C.

Il est peu probable que le terme soit ici péjoratif ; qualificatif habituel de Pittacos (bien que son titre réel soit aisymnète), il s'accorde bien à la toute-puissance d'Éros.

Comme il a adapté la théorie platonicienne des philiai à son propos, Plutarque ici adap te la législation de Solon, car cette obligation n'était faite que pour les mariages avec des filles épiclères, sans doute pour mieux assurer la naissance d'héritiers (cf. Sol. 20.4).

Le texte (18.763 E) est clair dans l'ensemble, mais pas dans le détail, où l'on n'a aucun

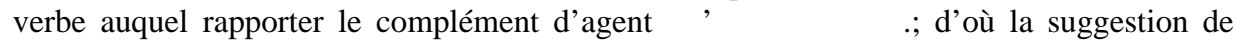
Barigazzi, 1986, d'ajouter après $\Sigma \dot{\lambda} \lambda \omega v o$ un participe qui serait tombé par un saut du même au même (mots homéotéleutes).

Sur ce sujet, voir la communication que j' ai faite au Colloque "Gott und die Gotter" de Gottingen en février 2005, "Gottlichkeit und Glaube. Persõnliche Gottesbeziehung im Spàtwerk Plutarchs» (Actes à paraître). 
sur les lèvres', selon l'expression d'Eschyle, il ne faut pas, je crois, le pas ser sous silence" (18.763 B). Ainsi, non seulement la divinité d'Éros est affirmée à chaque extrémité du développement , mais elle est en même temps solidement ancrée dans la tradition. Entre les deux, l'atmosphère sacrée, que préparaient les premières remarques de Protogène et Daphnée, s'accuse : ce sont les érastes qui défendent leurs aimés "comme des sanctuaires inviolables et sacrés ${ }^{97 " \text {; }}$ Alceste, femme privée du secours d'Arès, qui puise son héroïsme dans la "possession d'Eros " ; son exemple montre qu'Éros peut triompher même d'Hadès et les amants deviennent alors "les sectateurs et les initiés d'Éros99", participant à des Mystères supérieurs encore aux Mystères d'Éleusis. Cette atmosphère religieuse et mystique se prolonge et s'accentue avec l'évocation du cortège d'Éros célébré par la tradition et s'avançant au milieu de couples dont l'union "est comme portée par des ailes vers ce qui est le plus beau et divin

Elle prépare le développement sur l'Éros platonicien, lui-même objet d'un "dis cours sacré101", présenté comme un Éros "chaste et divin102", qui montre dans les beaux objets d'amour des miroirs "mortels des choses divines " lorsque ceuxci ont conservé "quelque trace du divin ${ }^{104 "}$ ". Alors - retour du thème suggé ré à la fin du premier mouvement - il est possible de retrouver le beau absolu et divin ${ }^{105}$ et le passage s'achève sur une nouvelle vision du Phèdre, beaucoup plus développée, où le "véritable amou reux...porte des ailes et célèbre conti-

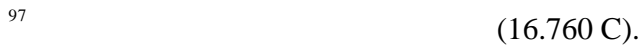

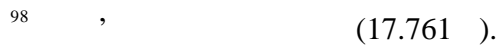

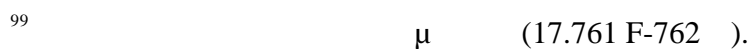

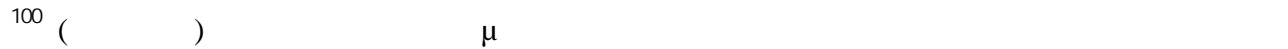
(18.763 F); le texte est plein de réminiscences du Phèdre qui vont jusqu'à des échos ver

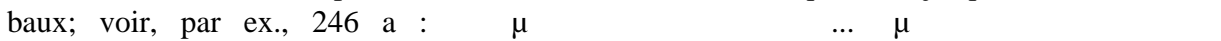

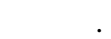

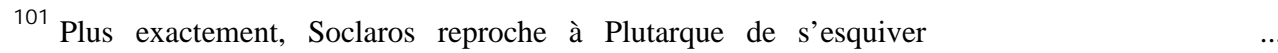

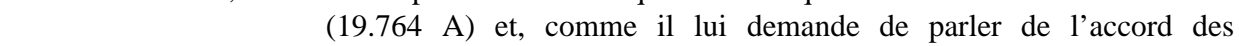
mythes égyptiens avec la doctrine platonicienne, c'est aussi bien les premiers qui pour raient être désignés comme "discours sacrés" ; mais en réalité Plutarque ne va parler que de Platon.

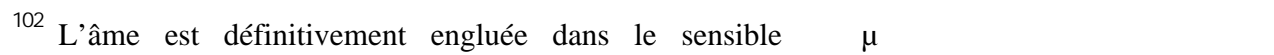

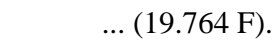

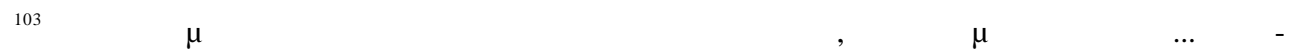
$v v \sigma 1$ (19.765 B).

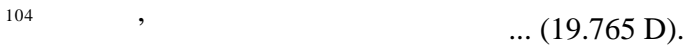

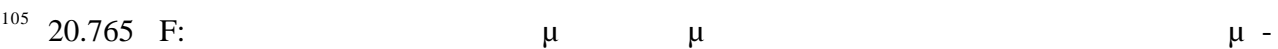

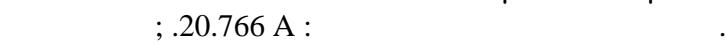


nuellement les mystères de son dieu qu'il escorte en dansant dans le ciel ${ }^{106}$ ".

Ainsi poussée à son point le plus extrême dans ces ultimes chapitres du discours central ${ }^{107}$, cette sacralité ne disparaît pas lorsque l'on revient à l'a mour conjugal : dans le même esprit que Daphnée, Plutarque fait de l'union charnelle, source de la philia, une "parti cipation commune à de grands mystè res ${ }^{109 "}$ et de ceux qui aiment les "hiérodules" du Dieu ${ }^{110}$. C'est que, après le discours central, aimer, èp âv, n'est pas autre chose qu'être habité par le Dieu ; de là l'affirmation que "dans le mariage, aimer est un plus grand bien qu'être aimé111" et le conseil donné à la femme mariée de "sacrifier à Éros" (23.769 D).

"Sacrifier à Éros" : le conseil, qui transpose la recommandation que Platon faisait au sombre Xénocrate de "qsacrifier aux Grâces", est ici pris au figuré. Mais le texte s'ouvre sur un sacrifice véri table : celui que la jeune épouse de Plutarque est venue faire avec lui à l'oc casion des fêtes d'Éros, les Érotideia , célébrées à Thespies, sans doute pour remercier le Dieu d'avoir réconcilié leurs familles ${ }^{110}$. Le dieu dont il est question dans tout le texte n'est ainsi pas seulement le dieu d'Hésiode, de Solon et de Platon, mais le dieu d'une tradition vivante. Rien n'est plus éclairant que la conclusion que tire Plutarque après s'être évertué à prou ver à Pemptidès la divinité d'Éros :

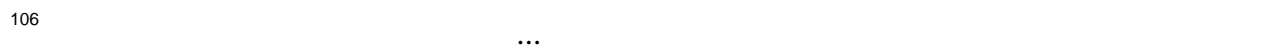

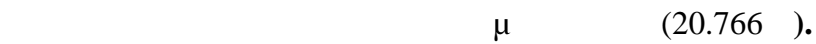

${ }^{107} \mathrm{Ou}$, du moins, ce qui constitue pour nous les ultimes chapitres, puisque nous n'avons pas la fin du discours ; néanmoins l'introduction qui nous est conservée montre que, si l'on quittait les sommets platoniciens, l'accent était toujours mis sur la puissance du dieu ("Éros, à l'instar des autres dieux, comme le dit Euripide, "se plaît à recevoir les hom mages des hommes' et s'irrite dans le cas contraire...”, 20.766 C).

$1083.750 \mathrm{C}$, cité supra, p. 81.

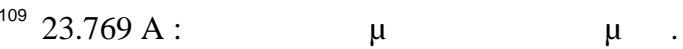

${ }^{110} 21.768$ B. Que cet élément ait ou non joué dans le choix de l'exemple, le fait que Camma soit une prêtresse d'Artémis et que la scène de mort se joue près de l'autel s'accorde bien avec cette atmosphère sacrée.

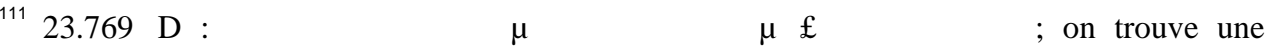
idée voisine dans le discours de Phèdre, expliquant que "l'amant est en effet plus divin que l'aimé puisque le dieu l'habite", (Banq. 180 b).

112 2.749 B le texte n'est pas très clair et l'on n'arrive pas à définir nettement ce qu'était "la" brouille (Plutarque emploie l'article défini) survenue entre les deux familles et si elle concer nait leur mariage, qui aurait, grâce au Dieu, triomphé de certains obstacles ; l'hypothèse est aussi séduisante qu'invérifiable. En tout état de cause, il faut souligner le rôle important que

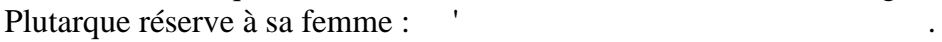


J'affirme donc en résumé que l'enthousiasme des amants n'est pas concevable sans un dieu, et qu'il n'a pas d'autre dieu comme protecteur et cocher que celui dont nous célébrons en ce moment la fête et à qui nous sacrifions ${ }^{113}$.

Située au cœur du discours le plus "théorique" de YÉrotikos, cette remar que invite à donner toute sa valeur à la mise en scène choisie par Plutarque.

Lui qui cède si souvent la parole à son frère Lamprias, il a fait ici de son fils le narrateur du dialogue, c'est-à-dire du fruit d'une union avec Timoxéna qui, au temps du dialogue, en était encore à ses débuts ${ }^{114}$. Le texte commence ainsi avec un jeune couple fraîchement marié 115 et s'achève sur le cortège nuptial d'un autre couple, celui d'Isménodore et de Bacchon, qui s'en va vers le sanctuaire du Dieu sous la conduite de Pisias ${ }^{116}$ : dans les deux cas, le Dieu apparaît bien comme le protecteur de l'union, celui que remercie le couple déjà uni, celui qui mène les opérations pour faire aboutir l'u nion du second. Jeune marié, Plutarque, qui se fera le défenseur du dieu, est pré senté par Pisias, non sans quelque ironie, comme "entièrement consumé et (assez) rempli de feu" (6.752 D) pour contaminer Daphnée, en passe de fondre pour Lysandra : il serait tentant de penser qu'ainsi, à travers Plutarque, c'est Éros qui s'exprime, de même que, selon lui, Platon n'a été que l'instrument des Muses ${ }^{117}$. Or les Muses jouent aussi un rôle important dans notre texte, puisque le dialogue se déroule en dehors de la ville, "auprès des Muses ${ }^{118 ", ~ l o i n ~ d e ~ l ' a g i t a t i o n ~}$ et des querelles, dans un lieu propice à la philosophie et sans doute à Éros : son ac tion n'est-elle pas présentée comme "dia métralement opposée" à celle d'Arès ${ }^{119}$ ? et n'est-il pas au contraire le compagnon des Muses, comme des Grâces et d'Aphrodite (15.758 C) ? Le cadre corres pondrait assez bien à l'état d'esprit que

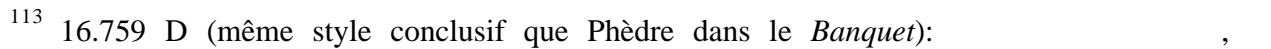

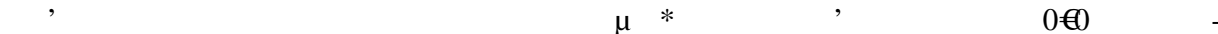

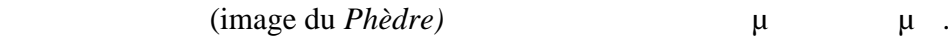

114 Et dont il est la preuve vivante de la pérennité.

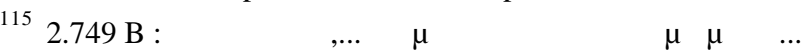

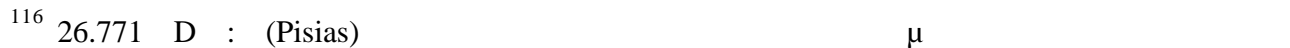

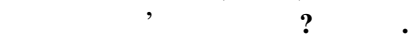

11719.764 A : $\tau \dot{\alpha} \quad \varepsilon v$ oưv $\dot{\alpha} \rho 1 £ \dot{\eta} \lambda \omega \quad \varepsilon \dot{\rho} \eta \dot{\varepsilon} v \alpha \quad \Pi \lambda \dot{\alpha} \tau \omega v 1, \quad \dot{\alpha} \lambda \lambda$ ov $\quad \delta \varepsilon \quad \tau \dot{\alpha} \hat{i} \quad \theta \varepsilon \alpha 1 \quad \tau \alpha \dot{\tau} \tau \alpha 1$ $\delta i \dot{\alpha} \Pi \lambda \dot{\alpha} \tau \omega v o \quad \ldots$

${ }^{118}$ La présentation du cadre commence et finit sur les Muses (1.748 F : "C'est sur l'Hélicon, auprès des Muses, alors que les Thespiens célébraient les Érotideia...”; 2.749 C : “ils allèrent camper auprès des Muses").

119 Ce qui pourrait peut-être expliquer la récurrence des images guerrières pour évoquer ce qui se passe en ville (cf. 2.749 C; $11.755 \mathrm{~B} ; 13.756 \mathrm{~A} ; 26.771 \mathrm{D}$ ). 
Daphnée prête à Solon lorsqu'il écrivait "Maintenant je me plais aux travaux de Cypris, de Dionysos et des Muses, qui apportent aux hommes la joie" : loin des tempêtes juvéniles des amours pédéras tiques, le Sage aurait alors trouvé refuge "dans la bonace du mariage et de la philo sophie" (5.751 E), une association qui peut paraitre paradoxale ${ }^{120}$ et qui cor respond au propre choix de Plutarque. C'est ainsi que, en l'absence de dédicace explicite, on peut lire le texte aussi bien comme une contribution de Plutarque à la fête du Dieu que comme un hommage à Timoxéna ${ }^{120} 121122$ * : les deux ne sauraient s' clure, puisqu'un mariage heureux est la manifestation concrète de la puissance et de la bonté du Dieu que Plutarque célèbre dans son discours central et qu'il mon tre à l'œuvre dans l'éloge final du maria ge. Pour mieux les cerner, il faut revenir aux liens du Dieu avec le pathos amou reux et approfondir sa puissance, qui en fait le maître, despotès, des amoureux, et sa bonté, qui en fait un maître, didaskalos, de sôphrosynè.

\section{Les bienfaits d'Éros (1) : la lumière de l'amour}

Patronnés par un Dieu, les symptômes de l'amour chantés par la poésie lyrique deviennent, comme dans le Phèdre, les signes mêmes de l'enthousiasme, les ger mes d'une union profonde et durable. Les images traditionnelles de lumière et de chaleur permettent ainsi à Plutarque de 'fondre' dans une même perspective psychologie de l'amant, désir platonicien et union conjugale. Daphnée évoque rapi dement d'entrée l'Éros conjugal, qui "ral lume par des naissances notre nature qui s'̧̧x́teint" (5.752 A); à quoi peut répondre dans un élargissement cosmique, la célé bration finale par Plutarque de "la nature (qui) montre que les dieux eux-mêmes ont besoin d'Éros" : ainsi "la Terre aime la pluie", "le ciel la terre" et "le soleil la lune" (24.770 A). Mais plus qu'à la fécon dité124 125, c'est aux effets sur l'âme que s'at tache Plutarque tout au long du texte. L'amour apparaît d'abord comme une brûlure : il enflamme Isménodore, Plutarque et Daphnée ; il est "l'enthousias-

On sait que la question de savoir si le Sage doit se marier était un sujet courant de dia tribe, mais si les Stoïciens, contre les Epicuriens, y répondaient par l'affirmative (voir les Diatribes de Musonius Rufus), il s'agissait surtout pour eux de remplir leur rôle social.

121

Ce qui est fatal à partir du moment où il a choisi un dialogue « au second degré »; pour faire une dédicace, l'auteur doit prendre directement la parole et s'adresser au dédicataire.

122 Ce que suggère Fl a c el ièr e, 1980, 39.

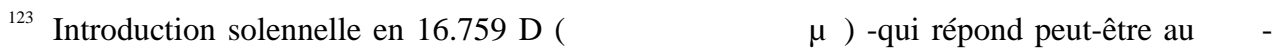

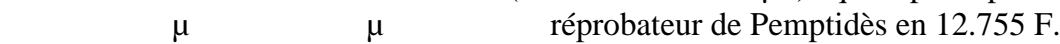

${ }^{124}$ On peut à nouveau relever dans ce jeu d'échos de la première à la troisième partie un effet de structure embrassée.

${ }^{125} 6.752 \mathrm{D}$ et $8.753 \mathrm{~A}$. 
me le plus chaud" (16.759 A), une "mania qui consume" (16.759 B) et inscrit les images de l'aimé dans la mémoire "comme si elles étaient peintes à l'en caustique et gravées avec du feu" (16.759 C); mais c'est surtout dans la partie expli citement consacrée aux bienfaits qu'on le voit comme éclairer de l'intérieur l'amant grâce à une remarquable utilisation des citations. C'est d'abord un éclat extérieur qui se manifeste : "de même qu'il arrive que 'quand le feu est allumé, la maison est plus chère à voir', ainsi, semble-t-il, un homme devient plus rayonnant ( $\varphi \alpha \iota_{1} \delta \rho \dot{~}$

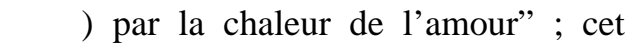
éclat est mal apprécié par certains, dont l'erreur permet à Plutarque d'intérioriser l'analyse : "mais il arrive à la masse quelque chose qui défie la logique ; s'ils voient de nuit une lueur dans une maison, ils pensent que c'est divin et l'admirent; mais quand ils voient une âme mesquine, basse et vile tout à coup s'emplir de senti ments nobles et élevés, d'honneur, de générosité, ils ne se sentent pas obligés de dire comme Télémaque : 'Assurément il y a un Dieu à l'intérieur' 127 ". Métamorpho se de l'amour, qui trouve sa pleine mesu re dans la description platonicienne des chapitres 19-20 et la comparaison-oppo sition avec le soleil.

Un premier rapprochement, superfi ciel, reprend les éléments déjà indiqués : le soleil comme Eros dispensent "rayonnement et chaleur ${ }^{128}$ ", l'un au corps, l'au tre à l'âme; mais le contraste s'accuse vite entre le monde physique et sensible d'un côté, le monde spirituel de l'intelli gible de l'autre. Eros n'est "lumière que des beaux objets 129 " et cette lumière, s'in tériorisant, se communique à la mémoi re ${ }^{130}$ qu'elle "rallume ${ }^{131}$ " en un phénomè-

126 La traduction littérale n'est pas très jolie, mais il me paraît important de mettre en valeur l'infinitif, qui limite pour l'instant la constatation à un regard extérieur.

127 18.762 D-E; cette conclusion est corroborée par l'exemple de Sapho, qui elle aussi exha

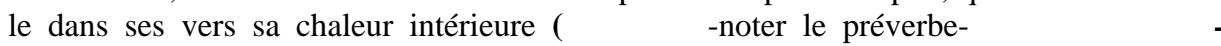

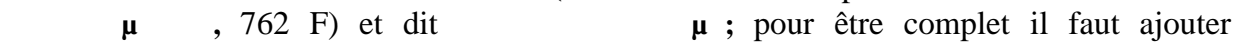
une curieuse comparaison de cette exhalaison enflammée avec les flammes vomies par

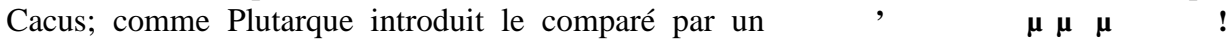
$\varphi \theta \dot{y} \gamma \gamma \varepsilon \tau \alpha$, Russel I 1997, 106, suggère qu'il y aurait peut-être une opposition entre le mythe et la réalité, pour accentuer la puissance du trouble érotique; on peut aussi penser à une opposition entre une manifestation purement physique et un phénomène de l'âme.

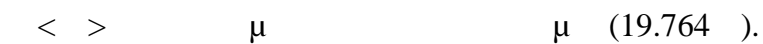

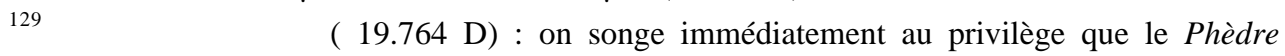
accorde à la beauté d'être "ce qui se manifeste avec le plus d'éclat et ce qui suscite le plus d'amour" (250 d).

${ }^{130}$ Dans cet élément aussi on peut voir un approfondissement de la première partie, où il n'était question que de l'image de l'aimé gravée dans la mémoire.

'Е

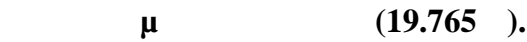


ne de réminiscence, interprété en termes d'optique, grâce à la comparaison de l'arc-en-ciel, comme un phénomène de réfraction : l'amant "est réfracté vers la beauté divine et intelligible" et le contact de la beauté physique ne fait qu'enflammer encore davantage son esprit ; c'est lui-même, tout entier, qui "se rallume à la beauté" intelligible ${ }^{134}$ et devant cette lumière s'emplit d'un désir dont Plutarque souligne l'activité .

Cet éclat extérieur de la beauté qui sus cite l'amour réapparaît dans la dernière partie consacrée à l'amour conjugal. S'attachant à démontrer qu'on peut aussi éprouver de l'amour pour les femmes, Plutarque cite expressément deux théories philosophiques de la naissance de l'a mour, la théorie épicurienne, qui ne fait l'objet d'aucun commentaire (21.766 E), puis la théorie platonicienne avec ses "belles et saintes réminiscences qui nous rappellent à la véritable, divine et olym pienne beauté de l'au-delà", plus dévelop pée, qui lui permet d'évoquer "les traces d'une âme éclatante136", qui transparais sent dans la beauté physique; mais il ajou te encore, sans la désigner nommément, la "manifestation (غ $\varphi \alpha \sigma 1)$ des disposi tions naturelles à la vertu" (21.767 B) chère à l'école stoïcienne et, pour soutenir qu'elle existe aussi chez les femmes, utili se à nouveau judicieusement la poésie et les images lumineuses; ayant approuvé les vers d'Eschyle : 'En voyant l'œil plein de feu d'une jeune femme / Je sais bien qu'à l'amour d'un homme elle a goûté", il fait ressortir l'illogisme qu'il y a à penser que les marques d'un caractère akolastos s'impriment sur le visage et à refuser que la lumière d'un caractère sôphrôn s'y montre . Mais il n'arrête pas là l'analy se; au contraire l'évocation des débuts de l'amour lui sert de prétexte à en suivre le développement et l'épanouissement.

Les bienfaits d'Éros (2) : l'épanouis sement de la sôphrosynè

Ainsi, de même que dans la partie 'mystique' centrale est dépeint à plu sieurs reprises le mouvement $\mathrm{d}$ de l'âme, annoncé en 17.762 A comme la victoire d'Éros sur Hadès , repris dans l'évocation d'Éros mystagogue en 19.765 A et détaillé à l'occasion des comparaisons avec $\Gamma$ “initiation” géomé-

\footnotetext{
${ }^{32}$ Avec probablement un jeu sur les mots $\alpha v \dot{\alpha} \quad v \eta \sigma \dot{\alpha} \dot{\alpha} v \dot{\alpha} \kappa \lambda \alpha \sigma 1$.

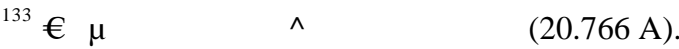

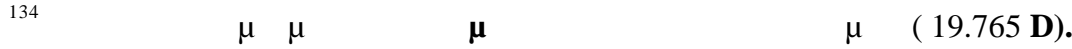

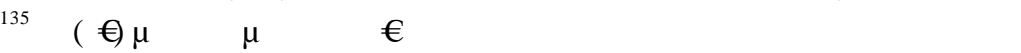
(20.766 B).

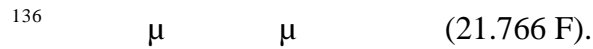

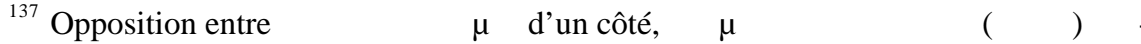
yo de l'autre $(21.767 \mathrm{~B})$.

${ }^{138}$ On trouve à la fois les mots $\dot{\alpha} v o \delta$ et $\dot{\alpha} \tau \rho \alpha \pi$.
} 
trique (19.765 A-B), puis avec l'arc-enciel (20.765 F) ou, mieux encore, à l'in térieur des peintures contrastées des bons et des mauvais amants (19.765 C-D et $20.766 \mathrm{~A}-\mathrm{B}$ ), de même la lente fusion des époux revient avec insistance dans les derniers chapitres, reprenant et expli citant à la fois la remarque que Plutarque avait faite dès sa première intervention sur les difficultés qu'une union avait à se maintenir sans les liens d'Eros ${ }^{139}$ et qu'il avait complétée un peu plus loin en évo quant les orages qui secouaient les cou ples juvéniles, malgré, ou plutôt à cause d'Éros ${ }^{140}$. Au commencement, expliquet-il ici, l'amour physique est certes sour ce de douleur, mais c'est pour permettre à la fois la conception d'enfants et l'u nion des époux : le texte parle de ${ }_{1} \xi_{1}$ (24.769 E), un "mélange", qui, au fil du temps, après la décantation des premiers troubles $^{141}$, va gagner en stabilité et devenir "l'union intégrale" des physi ciens $^{142}$, parvenant à une unité que seul Eros est susceptible de donner (24.769 F). On a là une variation, plus appuyée et couronnée par le beau mot d' $\dot{v} v \dot{c} \tau \eta$, de l'évolution déjà dessinée au chapitre 21 , où Plutarque expliquait, toujours selon le même schéma, que 'd'abord' celui que l'amour a envahi conservera les principes du 'mien' et du 'non-mien' et que ne parviendront à la véritable communauté que "ceux qui bien que séparés physiquement, de force, réunis sent et fondent leurs âmes, ne voulant ni ne pensant plus être deux ${ }^{143}$ ".

L'union ainsi scellée n'a pas qu'une dimension affective; elle a aussi une haute valeur morale. C'est pourquoi, après avoir évoqué cette fusion, gage de stabilité, Plutarque introduit aussitôt comme second point ${ }^{144}$ ce que nous tra duisons, faute de mieux, par "fidélité

$1396.752 \mathrm{C}$ où le verbe employé est $\sigma u v \varepsilon \dot{\chi} \dot{\varepsilon} \sigma \theta \alpha \mathrm{l}$ (le préverbe est important).

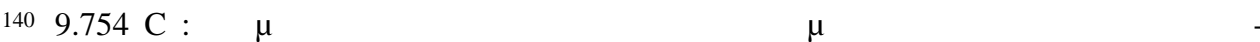

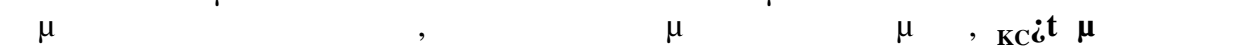

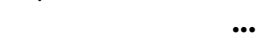

141 Évoqués par une double comparaison, avec les liquides ( $\dot{\omega} \sigma \pi \varepsilon \rho \dot{v} \gamma \rho \dot{\omega} v \pi \rho 0 \quad \dot{\alpha} \lambda \lambda \eta \lambda \lambda \alpha$

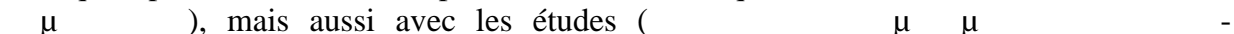

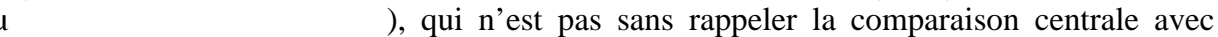
la géométrie (24.769 E-F).

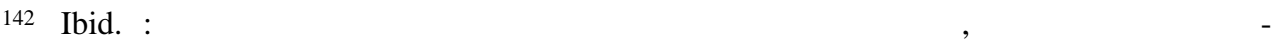

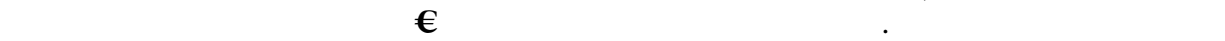

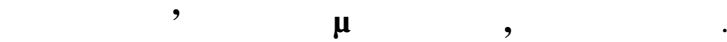

14321.767 D-E : le détail du texte est malheureusement rendu incertain par une lacune; Zuntz

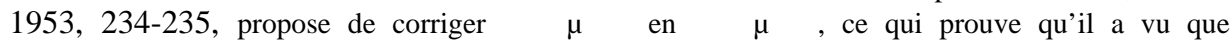
les deux passages se faisaient écho, mais il ne daigne pas expliquer pourquoi il veut intro duire dès le premier passage la notion d'unité, au demeurant déjà très présente dans les pré

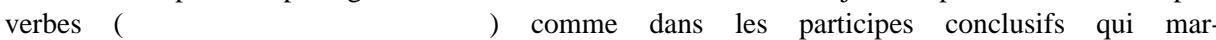
quent, en une belle progression, la volonté, puis la conviction de ne plus faire qu'un.

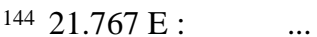


réciproque", mais qui est exprimé en grec par le mot sôphrosynè. Ayant jeté le thème en tête de phrase, il en développe une première forme : celle qui résulte de la contrainte sociale ${ }^{145 * 147}$, et qui est évi demment une forme inférieure; on s'at tendrait à trouver ensuite la définition d'une seconde forme, supérieure ${ }^{146147}$. Or il rompt la construction et cette anacolu the met en relief la haute valeur morale d'Éros, dont les effets extraordinaires sont appuyés par une tournure consécutive : "Mais Eros a en partage tant de maîtrise de soi, de décence et de fidélité que, même si d'aventure il atteint une âme akolastos, il la détourne de ses autres amoureux et, détruisant son orgueil et bri sant son insolence pour lui apporter pudeur, silence, calme et bonne tenue, ne la rend plus attentive qu'à un seul" (21.767 E). Exclusivité de l'amour enco re, mais aussi métamorphose, remarqua ble entre toutes, puisque chacune des qualités nouvelles s'oppose au dérègle ment initial ; métamorphose qui rappelle et prolonge, dans son style même, les métamorphoses des chapitres 17 et 18 consacrés aux bienfaits d'Éros : il y était question de générosité, de bonté données par l'amour aux âmes les plus mesquines, mais l'évocation avait très vite quitté le plan éthique pour revenir au plan spirituel et évoquer la dépossession de soi, l'arra chement au quotidien, comme preuve de la divinité d'Éros ${ }^{148}$. On a donc ici une sorte de complément où est exaltée la plus belle des vertus morales qu'Éros puisse donner, une vertu qui est précisé ment l'antithèse de la passion débridée à quoi ses adversaires voudraient le rédui re $^{149}$. Quelle meilleure réponse que d'en faire la source même de la sôphrosynè ?

Or ces rapports privilégiés avec la vertu si chère à Platon permettent aussi de tisser des liens plus étroits avec la partie centrale, où elle joue un rôle important ${ }^{150}$; ainsi, au niveau métaphy-

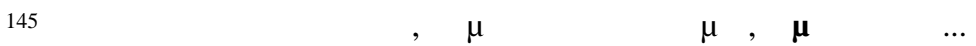

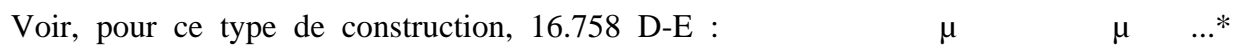

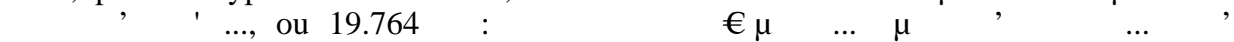

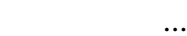

147 'Е $\rho \omega \tau 1 ~ \delta \varepsilon \ldots$.

$148 \mathrm{La}$ rupture se produit en $18.762 \mathrm{E}$, avec une introduction sans équivoque : Ekعivo $\delta \backslash$ \.ov่ $\delta \alpha 1$ óviov; le premier temps reste dans Torbe éthique et Plutarque y évoque, dans un portrait qui ressemble à celui de notre passage, $\mathrm{T}$ amant plein de morgue, qui, à la vue de son aimé, et de lui seul, voit "son audace brisée et réduite en miettes le fierté de son âme" mais l'accent est mis davantage, semble-t-il, sur la puissance d'Éros que sur la valeur mora le, et, surtout, l'introduction de Sapho qui suit amène aussitôt un changement de plan.

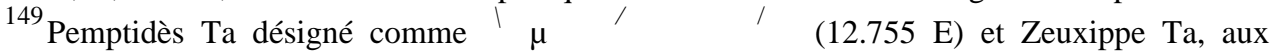

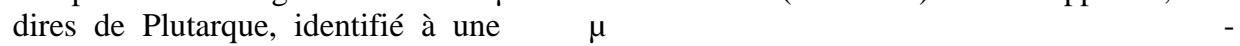

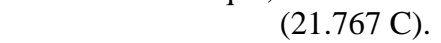

150 Alors que, dans le feu de la polémique, Plutarque a, dans son premier discours, à propos d'Isménodore, rejeté de la manière la plus expéditive les femmes sôphrones comme invi vables (voir supra, p. 70). 
sique des retrouvailles avec le Beau comme au niveau éthique de l'union conjugale, l'accent est mis sur elle. Le mystagogue platonicien est un Eros "divin et sôphrôn" (19.764 F) et les véritables amants sont ceux qui, "par le moyen d'un raisonnement sôphrôn accompagné de pudeur, absolument comme pour un feu, ont enlevé ce que cette passion pouvait avoir de furieux et n'ont conservé à l'âme que son éclat et sa lumière ainsi que sa chaleur" (19.765 B). On voit bien ici com ment s'articulent ardeur et sagesse : ayant établi désormais la divinité d'Éros et écar té le risque de le confondre avec une pas sion furieuse, Plutarque peut revenir à la forme négative de la mania, à laquelle pensait Pemptidès et que lui-même avait rapidement présentée en $16.758 \mathrm{D}$, pour ne traiter ensuite que des formes positives $\mathrm{du} \quad$ Phèdre; réapparait alors l'opposition traditionnelle entre bons et mauvais amants $^{151}$, ou, pour le dire comme lui, entre les polloi, qui, à l'instar d'Ixion, cherchent à empoigner un objet qui se dérobe et ne peuvent connaître qu'un plai sir mêlé de peine, et l'amant euphyès et sôphrôn, qui y trouve l'occasion d'une élévation spirituelle et finit par rejoindre le cortège du Dieu (20.766 A).

Cette opposition se retrouve dans la dernière partie : il n'est pas question de condamner en bloc la beauté ou l'amour des femmes en les associant systémati quement à une forme intempérante, et donc fausse, d'amour, comme Protogène et sans doute Zeuxippe, mais il faut, sur chacun de ces points, distinguer la femme akolastos de la femme sôphrôn. Ainsi la première utilise sa beauté pour séduire et entraîner au seul plaisir, tandis que la seconde y voit un moyen de s'attacher son mari et de susciter de sa part les senti ments durables et profonds que sont Veli nola et laphilia (23.769 C). Aux deux ce pendant Eros peut accorder ses bienfaits et si, comme on l'a vu, il peut transformer même une âme dissolue ${ }^{153}$, la femme

151 Qu'on ne trouve pas encore à proprement parler dans le passage que je viens de citer, puisque, à ceux qui ont su régler le feu de l'amour, s'opposent ceux qui "cherchent à étein dre de façon brutale et déraisonnable cette passion" -le heurt voulu entre pathos et alogôs, qui fait de la lutte contre la passion, et non de la passion, quelque chose de contraire à la rai son a été relevé par Babut, 1969, 325, n.4 ; faut-il voir les Stoïciens derrière ce groupe ? La suite en tout cas semble plutôt incliner vers une réfutation de l'épicurisme : au lieu d'une douce chaleur, ils ne réussissent en effet qu'à s'emplir de fumée et de trouble (Plutarque file l'image avant de revenir à une notion plus psychologique, antithèse de l'ataraxie) ou bien ils se ruent vers des plaisirs ténébreux (toujours l'image de la lumière) et illégitimes (ce qui semble évoquer la Venus volgivaga de Lucrèce, qui doit soulager sans créer de liens amou reux durables, source de tourments : cf. De rerum nat. IV. 1049-76).

${ }^{152}$ C'est dans cet esprit en tout cas que semble répondre Plutarque (voir en part.23.769 B), ce qui ne prouve rien, puisqu'il ne répond pas à la précédente objection de Pemptidès dans l'esprit moral dans lequel elle a été posée, mais l'infléchit dans le sens théologique qui l'intéresse.

${ }^{153} 21.767$ E, cité supra, p. 91, qu'il illustre par l'exemple de Laïs. 


\section{L'Érotikos : un éloge du Dieu Èros ? Une relecture du dialogue de Plutarque 93}

chrèstè et sôphrôn se doit aussi de "sacri fier à Èros", car "dans le mariage aimer vaut mieux que d'être aimé" (23.769 D).

Dans cet esprit, l'union chamelle cesse d'être la marque définitive de l'infériorité de l'amour des femmes, esclave du plai sir : il est important sans être l'essentiel de la relation conjugale et rien ne le dit mieux peut-être que la manière dont Plutarque, au long de son texte, redéfinit les rapports d'Éros et d'Aphrodite. Ils sont inséparables, mais si Daphnée, sui vant la vision la plus traditionnelle, fait d'Éros le serviteur d'Aphrodite ${ }^{154}$, Plu tarque tend à inverser les rapports; sans doute il souligne qu'on ne peut injurier Èros sans toucher à Aphrodite (13.756 F); mais il explique aussi que la fécondité qui vaut à la déesse tant d'éloges "est ergon d'Aphrodite, mais parergon d'Éros lors qu'il assiste Aphrodite" (13.756 E) : un préfixe fait toute la différence, et ce qui est œuvre essentielle d'Aphrodite n'est plus qu'œuvre secondaire pour Èros, qui apporte bien plus, la communion spiri tuelle qui fait tout le prix de l'amour. Le discours central insiste ainsi sur la né cessaire présence d'Éros pour donner de la valeur aux œuvres d'Aphrodite ${ }^{155}$ et utilise finalement les assimilations égyp tiennes Isis-Aphrodite-Lune d'un côté, Osiris-Éros-Soleil de l'autre, pour con firmer la supériorité du second ${ }^{156}{ }^{157}$. Après quoi, la hiérarchie établie, il peut repren dre la formulation de Daphnée et évo quer, dans ses réflexions sur le mariage, la nécessaire présence d'Aphrodite, en s'indignant : "Qui supporterait de voir qu'on outrage Aphrodite en prétendant que, si elle se joint à l'amour et l'assiste, elle empêche la philia de naître ?" (23.768 E). L'union des époux est au contraire germe de philia, car —et le texte à nouveau s'efforce, avec une belle image de germination, d'évoquer la communauté qui se crée- "si le plaisir est peu de chose", essentielles sont "l'estime, la complaisance, l'affection réciproques et la fidélité qui s'épanouis sent chaque jour à partir de ce germe 157 *". Dans cette liste de nobles sentiments, un sort particulier doit être réservé d'abord à 'la complaisance' : le beau mot de $\chi \dot{\alpha} \rho 1$ si malaisé à traduire sans l'affadir, avait été introduit par Daphnée comme l'instrument de l'union conjugale, mais défini, de façon limitée, comme "l'acquiescement de la femme au désir de l'homme" (5.751 D) et repris

1545.751 B : “Comment s'agirait-il d'Éros, si Aphrodite est absente, elle qu'Éros, par la volonté des dieux, a pour rôle d'assister et d'entourer en participant à ses honneurs et à sa puissance dans la mesure où elle le lui permet ?"

155 Outre $13.756 \mathrm{C}$ cité, voir $16.759 \mathrm{E}$ (texte conjectural, mais dont le sens parait clair) et $759 \mathrm{~F}$.

$15619.764 \mathrm{D}$ : (la lune) "par elle-même est dépourvue de puissance et de lumière, à moins que le soleil ne l'éclaire, —comme l'est Aphrodite, à moins qu'Éros ne l'assiste”.

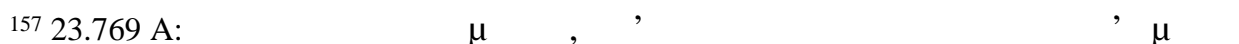

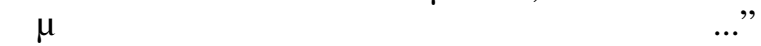


sans commentaire par Plutarque comme le moyen, avec la $\pi \varepsilon 1 \theta \dot{\omega}$ amoureuse, de cimenter la communauté des époux (6.752 C) ; en l'insérant dans cette liste et en le complétant par $\alpha \lambda \lambda \eta \lambda \omega \nu$, il in siste bien sur l'idée de réciprocité inhé rente à cette notion et si importante dans les relations amoureuses ${ }^{159}$, lesquelles débouchent — second mot à relever ${ }^{160}$ _ sur la $\tau \tau i \sigma \tau \imath \cdot$ Attachement fidèle et indé fectible dont Empona sera l'exemple le plus éclatant ${ }^{161}$, elle résume à elle seule toute la valeur morale d'un amour qui ne parvient à sa plénitude que par la durée.

\section{Les bienfaits d'Éros (3) : les occur rences d'eumenès}

Tels sont les dons d'Éros, dont la bienveillance est soulignée par Plutar que dans chacun des types de dévelop pement qu'il lui consacre : l'eupéneia est une des rubriques de l'éloge reli gieux central ${ }^{162}$, forme que prend l'attri but divin $\dot{\alpha}^{\prime} \dot{\omega} \varphi \dot{\varepsilon} \lambda \alpha \alpha^{163}$, en parfaite har monie avec la tonalité de la première partie du discours où Plutarque souli gnait, à l'intention de Pemptidès, la "monstrueuse ingratitude" qu'il y avait à mettre en doute ainsi la bonté divine ${ }^{164}$; la partie platonicienne prolonge cette idée en présentant Éros, sauveur et mé decin, sous les traits d'un mystagogue bienveillant ${ }^{165}$ et on la retrouve encore dans l'éloge du mariage, où Plutarque recommande à la femme sage de "sacri fier à Éros, afin que, installé au foyer, il

${ }^{158}$ Il faut faire porter à l'évidence le génitif à la fois sur les trois substantifs.

${ }^{159}$ Sur le problème posé par la réciprocité amoureuse aux penseurs de l'Antiquité, voir Cal ame, 1997, en part, ch.l "L'Éros des poètes méliques", 23-52 et, pour Platon, Hal per in, 1986.

${ }^{160}$ On pourrait aussi s'arrêter sur $\tau ı \dot{\eta}$, car l'estime dans le mariage est intervenue aussi dans le premier discours de Plutarque, qui répondait aux inquiétudes de Pisias de voir Bacchon réduit à un rôle secondaire par une épouse plus riche et plus âgée (voir 9.754 A).

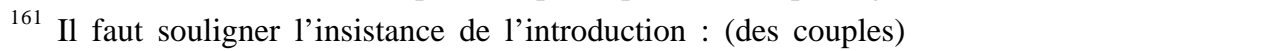

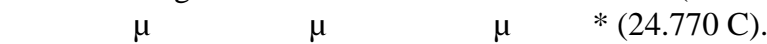

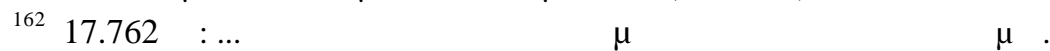

${ }^{163}$ Présenté avec la $\delta \dot{v} \alpha \alpha 1$ en 16.759 D; il faut noter que les attributs divins sont ordinai rement au nombre de trois (cf. De repugn. $1051 \mathrm{~F}$ et Arist. 6.3) : la "disparition" de l'in corruptibilité, qui s'intégre dans une vision cosmologique, laisse seuls présents deux attributs qui mettent la divinité en relation avec les hommes.

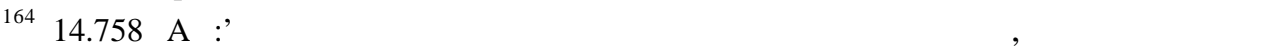

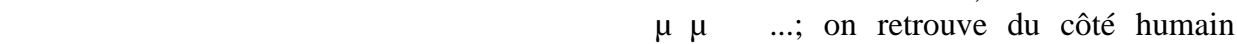
- conformément à sa valeur de réciprocité- la notion de charis, présente dans l'intro duction citée à la note 162 .

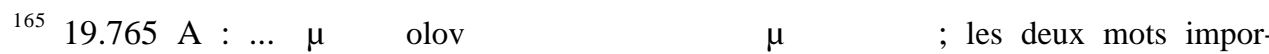
tants encadrent l'expression et la présence secourable du dieu est encore accentuée par le verbe. 
veille avec bienveillance sur son maria ge ${ }^{166 " . ~ E n f i n, ~ s i ~ l ' o n ~ q u i t t e ~ l a ~ p a r t i e ~ d i a l o-~}$ guée pour considérer l'action, là aussi, dans les dernières lignes, Éros apparaît comme celui qui a guidé avec bien veillance les événements, le metteur en scène, pour ainsi dire, du mariage d'Isménodore et de Bacchon ${ }^{167}$, le respon sable, sans doute, de la conversion specta culaire de Pisias, signe incontestable d'une puissance que Plutarque s'en va adorer ${ }^{168}$ et qui est le second attribut essentiel de la divinité. C'est ainsi que, à la fin de son dis cours central, revenant après l'évocation du telos platonicien de l'amour à des his toires concrètes, il introduit la punition de Gorgo en soulignant que, si Éros est "le plus bienveillant (des dieux) à qui l'ac cueille comme il convient, il pèse de tout son poids sur ceux qui, dans leur infa tuation, le repoussent ${ }^{169}$ '. Particulièrement éclatante dans sa vengeance, cette puissan ce néanmoins irrigue tout le texte et mérite de conclure l'étude de l'éloge d'Éros.

\section{La puissance universelle d'Éros}

Cette puissance se manifeste d'abord par la manière dont Éros s'empare des amants et, de même que la mention de son eumeneia revient dans tous les passages importants, de même on peut relever les occurrences des mots de la famille de lambanein. Dans l'histoire d'amour thespienne d'abord, Isménodore n'a pu agir que parce qu'une inspiration divine s'était emparée d'elle ${ }^{170}$ : elle est la pre mière illustration de la mania divine, qui va servir de preuve dans le discours cen tral, d'abord de la divinité d'Éros, puis de sa puissance. Les vers de Sapho sont ainsi commentés comme marques d'une $\theta \varepsilon o \lambda \eta \psi i \alpha$ évidente (18.763 A) et l'illo gisme de l'amour qui fait que le même objet consume l'un et laisse l'autre de marbre est aussi le fait du Dieu qui choi sit qui il veut toucher : $\beta \ddot{i} \lambda \eta \tau \tau \tau \alpha 1 \delta^{\prime \prime}$ elç

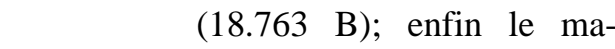
riage, à son tour, ne peut que réussir, si le Dieu s'en empare171.

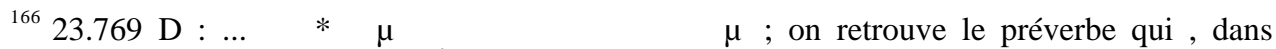

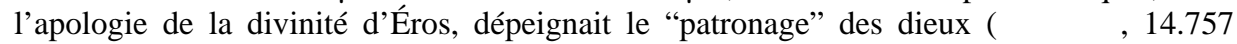

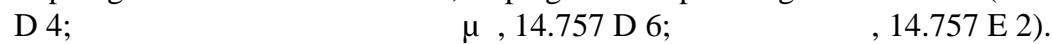

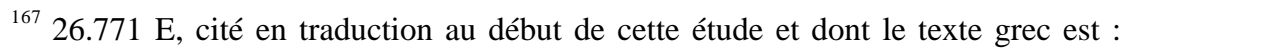

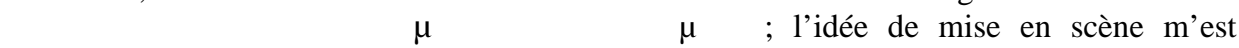
inspirée à la fois par l'aspect théâtral des événements (l'enlèvement, le cortège final, qu'on a pu comparer à un kômos) et par la comparaison avec le De Genio, où la Fortune semble mener le jeu et multiplier à dessein les péripéties (596 D).

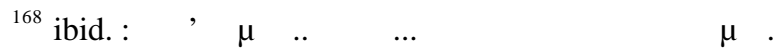

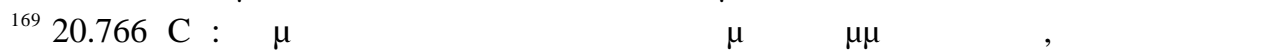
$\dot{\alpha} \pi \alpha v \theta \alpha \delta 1 \sigma \alpha$ ह่vo1.

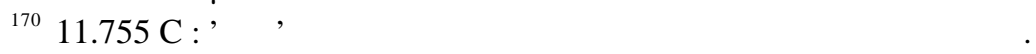

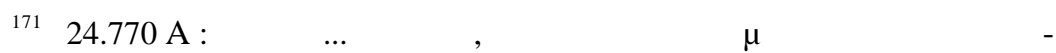

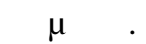


Dieu qui s'empare de ceux qui lui sont voués ${ }^{172}$, il se fait non seulement leur guide, celui qui "se met à leur tête pour les protéger", si l'on veut glo

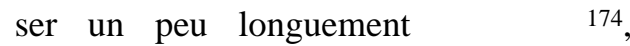

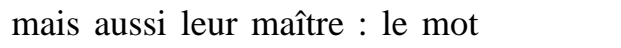
apparaît dans la réplique à Pemptidès, qui montre que la philia formatrice de l'amour des garçons "n'a pas comme guide et maitre d'autre dieu que le com pagnon des Muses, des Grâces et d'Aphrodite, Éros" (15.758 C) et insiste sur l'idée que la forme 'érotique' de la

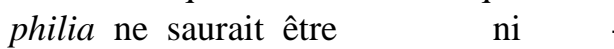

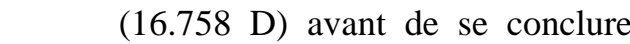
sur l'image triomphale d'Éros proclamé unanimement par Hésiode, Platon et Solon "roi, archonte et harmoste" $(18.763$ E). Il revient surtout dans la célébration de l'indéfectible fidélité de ceux qu'inspire Éros : "Nous savons aussi que d'humbles servantes fuient les embrassements de leurs maitres et que de simples particuliers méprisent des reines, lorsqu'ils ont installé Éros comme maître dans leur âme ${ }^{175 "}$; l'op position ainsi suggérée entre les maîtres temporels et le maître suprême qu'est Éros se développe dans une comparai son avec le dictateur romain dont la nomination suspend toutes les autres archai : "de même, ceux dont Éros s'est rendu maitre' ${ }^{176}$, sont désormais libérés et affranchis des autres maitres et archontes, comme des hiérodules". Les rapports de force dans le mariage qu'é voquait Pisias pour stigmatiser la volonté de puissance d'Isménodore avaient amené Plutarque à évoquer ironique ment les puissants qui s'étaient laissé subjuguer par d'humbles esclaves, déve loppant l'histoire de Sémiramis, présen tée comme "la servante concubine d'un esclave né dans le palais" du "grand roi" Ninos, et qui, malgré une distance sociale si considérable, "réussit à le domi ner et le méprisa ${ }^{179}$ au point de lui demander de la laisser un seul jour s'as seoir sur le trône ceinte du diadème et diriger les affaires." Elle en profita alors pour le tuer et s'emparer du pouvoir. La faiblesse de Ninos l'a fait succomber à

${ }^{172}$ Ce que suggère peut-être l'emploi dans certains passages de $\varepsilon \rho \omega \tau$ tıkó plutôt que de $\dot{\varepsilon} \rho \dot{\omega} v$ $\tau \varepsilon$; voir en part. 20.766 B, mais la chose n'est pas sûre, ni même, peut-être, constante.

173 À nouveau l'idée apparaît d'abord dans la démonstration initiale du discours central (

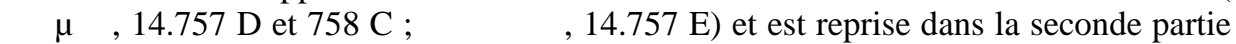

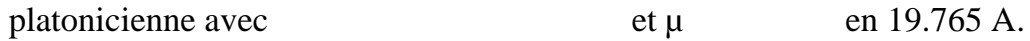

${ }^{174}$ Qui apparaît en 16.758 D et 759 D.

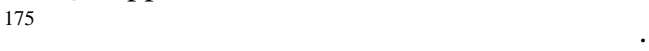

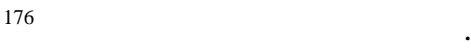

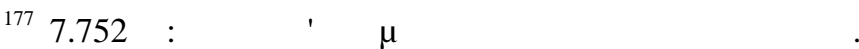

178 Tous les détails sont choisis pour abaisser l'une et rehausser l'autre; les origines habi tuelles de Sémiramis sont plus glorieuses; voir Diodore, II.4.

179 On retrouve les mêmes verbes pour Cléopâtre vis-à-vis d'Antoine (Ant. 26.1). 
la force d'Éros ${ }^{180}$, mais dans des âmes mieux trempées, cette force donne toute sa mesure. C'est ainsi que, dans la com paraison de la puissance d'Éros et d'Aphrodite, Plutarque montre d'abord comment Aphrodite privée d'Éros ne retient pas les époux de prostituer leurs épouses à plus puissant qu'eux pour servir leurs intérêts, puis, à l'inverse, com ment Éros seul pousse les érastes à s'op poser aux tyrans pour défendre leurs aimés, et donc à défier une puissance tem porelle à laquelle ils n'osent rien rétorquer en toute autre circonstance, et il cou ronne sa démonstration en évoquant Alexandre, le plus puissant des rois, qui s'inclina devant Éros et laissa à ses com pagnons, quel que fût son propre désir, les musiciennes dont ils étaient épris. Avec le passage du chapitre 21 dont cette analyse est partie, le thème atteint son akmè et Plutarque, après avoir évoqué les 'hiérodules' d'Éros, enchaîne : "Une femme noble qu'Eros a unie à un homme loyal supporterait plus facilement l'étreinte d'ours ou de serpents que le contact d'un autre homme partageant son lit" (768 B). Une telle préférence dit assez la violence des sentiments ; elle indique aussi que la fidélité amène à affronter la mort.

Puissance universelle, dieu dont la dimension cosmique apparaît bien dans les interventions de Plutarque, Eros apparaît finalement comme plus fort que la mort, et sur ce point aussi l'accord se fait entre le mythe, la théorie philoso phique et la réalité historique. Le thème est introduit par l'exemple le plus célè bre qui soit : celui d'Alceste ${ }^{185}$; son sort, comme celui de Protésilas ou d'Eurydi ce, montre que seul Éros se fait obéir d'Hadès; que seuls les amants peuvent remonter à la lumière ; du registre my thologique, on passe alors au domaine de la philosophie, et c'est, aux chapitres 19-20, l'évocation d'Éros mystagogue, où l'accent est mis sur Гanamnèsis et la

${ }^{180}$ Il illustre ainsi ce que note Plutarque en $19.764 \mathrm{C}$, parmi les ressemblances du soleil et d'Éros : “enfin, pas plus qu'un corps qui n'est pas exercé ne peut supporter sans dom mage le soleil, une âme sans éducation ne peut supporter Éros; le corps comme l'âme sont pareillement troublés et malades et ils en accusent la puissance du Dieu au lieu de s'en prendre à leur propre faiblesse."

18116.759 F-760 B : Gabba cède sa femme à Mécène et Phaÿllos envoie la sienne au roi Philippe.

$18216.760 \mathrm{~B}-\mathrm{C}$ : (il est impossible de trouver un éraste qui ait prostitué son éromène) $\pi \mathrm{O} \theta \varepsilon v$

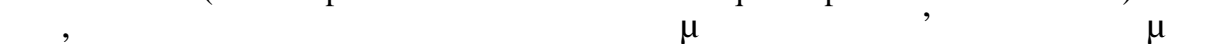

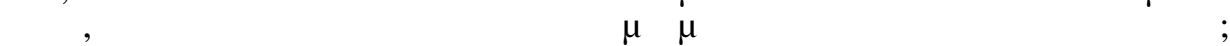
(noter l'abondance des préverbes $\dot{\alpha} \nu \tau 1-)$.

${ }^{183}$ La traduction française affaiblit quelque peu le verbe grec $\sigma 0 \gamma \kappa \rho \alpha \theta \varepsilon i \sigma \alpha \delta$ ' 'E $\rho \omega \tau$ * * .

${ }^{184}$ En 13.756 E-F et en 24.770 A-B (c'est-à-dire dans les deux éloges, du Dieu et du mariage).

18517.761 F-762 A : on la trouve dans le discours de Phèdre du Banquet comme dans les diatribes sur le mariage de Musonius Rufus (frg XIV). 
remontée de l'âme, et qui s'achève par une opposition entre "les hommes et les femmes attachés au corps et au plaisir, qu'on appelle indûment 'erôtikoi', qui, après la mort, reviennent hanter les chambres nuptiales 186" et "l'amoureux véritable, (qui) une fois dans l'au-delà et ayant fréquenté la beauté, comme il est juste, porte des ailes, célèbre les mystè res de son dieu et ne cesse plus de dan ser autour de lui et de l'escorter là-haut jusqu'au moment où il revient aux prai ries de la Lune et d'Aphrodite et après s'y être endormi commence une nouvel le naissance"(20.766 B). À cette vision eschatologique $^{187}$ succède, sur terre, l'héroïsme de Camma et d'Empona, deux Galates qui montrent que la puis sance d'Eros s'impose à tous, Grecs et barbares. La première, en butte aux avances de Sinorix, "un des chefs gala tes les plus puissants", qui n'hésite pas à tuer Sinat, son époux, dans l'espoir de l'obtenir, sacrifie sa vie pour venger ce dernier et le rejoindre en une scène pathétique et grandiose, où, devant l'au tel d'Artémis dont elle est la prêtresse, et après avoir bu et fait boire à Sinorix un poison, elle invoque Sinat, et, comme un vivant, l'implore de la "prendre avec

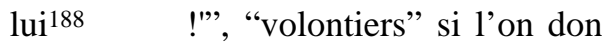
ne à ce participe son sens le plus banal, "avec joie" si l'on tient compte de sa valeur première, et cette joie s'accorde rait bien avec la résolution joyeuse que Camma elle-même montre face à la mort $^{189}$. L'histoire d'Empona, beaucoup plus longue à tous égards, par l'étendue du récit comme par la durée des événe ments qu'il narre, tisse des liens encore plus complexes entre l'amour, le pou voir et la mort : pour Sabinus, son époux compromis dans le soulèvement de Civilis et qui se fit passer pour mort, pendant plus de sept mois "elle vécut à peu de chose près comme dans le royau me d'Hadès" (25.771 A), tenta par une équipée rocambolesque à Rome d'obte nir son pardon ${ }^{190}$, mit au monde "deux lionceaux" avant d'affronter la mort et Vespasien en une scène qui répond à celle de Camma, proclamant qu'“elle avait mené dans l'obscurité souterraine une vie plus douce que lui sur son trône" (771 C). Si Eros au niveau métaphy sique sauve les amoureux de l'Hadès et leur permet de rejoindre la Beauté divi ne et absolue, sur terre, dans l'histoire

${ }^{186}$ Ce passage transpose Phaedo 81 a-b.

187 Qui associe Phèdre et mythe d'Er; voir aussi De Facie 944 C sq..

${ }^{188}$ Nuv ôe kó $1 \sigma \alpha i \quad \beta \chi \alpha \iota \rho \omega v$, moyen auquel s'oppose le passif réservé à Sinorix mou

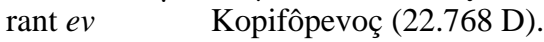

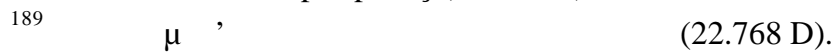

${ }^{190}$ Pour ce faire, elle le déguise, comme Phaÿllos avait déguisé sa femme, mais pour l'en voyer à Philippe. 


\section{L'Érotikos : un éloge du Dieu Èros? Une relecture du dialogue de Plutarque 99}

humaine, il transforme une vie compara ble à l'Hadès en vie heureuse et punit l'empereur qui, contrairement à Alexan dre, n'a pas respecté les amoureux en anéantissant toute sa famille ${ }^{191}$ : on peut reconnaître là, concluant le mouvement final, une des formes de la némésis que Plutarque évoquait à la fin du discours central en introduisant l'histoire de Gor go, avec, en plus, une forte insistance sur l'historicité du fait et les relations de Plutarque avec le fils d'Empona, "Sa binus (qui) est venu tout récemment chez moi à Delphes." (771 C).

En guise de conclusion : la polypho nie d'Éros

M. Fusillo, dans une intéressante étude sur le roman, a souligné ce qu'il appelle "la polyphonie d'Eros ${ }^{192}$ ", atti rant ainsi l'attention sur Гutilisation de la tradition littéraire par les romanciers et sur l'importance dans leurs œuvres de l'intertextualité. Ce dieu 'polyphonique' rencontre ici un auteur philosophos et philologos qui lui-même donne volon tiers à ses dialogues une forme polypho nique. Il en résulte une oeuvre com plexe, où Plutarque trouve l'occasion à la fois de célébrer le bonheur du maria ge, de défendre la religion ancestrale, et, dans le prolongement de celle-ci, d'évo quer le telos platonicien : de là proba blement le rôle écrasant qu'il s'est donné dans le texte, en parfaite contra diction avec l'âge qu'il était censé avoir à l'époque de la visite à Thespies. Tout ce qui pouvait compter pour lui, dans sa vie et dans sa pensée, éthique, religion et métaphysique, se trouvait réuni par la figure d'Éros, non pas la passion funes te des Tragiques, ni l'enfant joueur de l'Anthologie grondé par Aphrodite, mais le dieu fêté à Thespies et magnifié par Platon, le dieu toujours présent dans la vie des hommes : dieu primordial $\pi \dot{\alpha} v$ $\tau \omega \nu \pi \rho \gamma^{\wedge} v \varepsilon \dot{\varepsilon} \sigma \tau \alpha \tau o$, dieu qui confère aux couples leur durée, dieu qui permet aussi de braver et dépasser la mort. La complexité d'Éros répond finalement à la complexité même de la vie, qui fait du même être l'époux de Timoxéna, le père d'Autoboulos, le prêtre d'Apollon et un philosophe platonicien : une présenta tion est forcément analytique et ces 'strates' ne peuvent s'effacer totalement dans la contexture du dialogue, mais à travers les jeux d'échos, la récurrence des thèmes et leurs variations, la place centrale réservée à l'éloge du Dieu et à ses bienfaits eschatologiques, qui appa raissent comme l'horizon de la fusion des époux, Plutarque rend sensibles la manière harmonieuse dont il vivait sa religion et sa philosophie sans avoir le sentiment qu'il existait entre les deux une solution de continuité, la volonté qui

${ }^{191}$ Plutarque ne dit pas explicitement que c'est Erós qui sème la mort dans la dynastie flavienne, mais seulement que l'empereur fut puni, selon un procédé courant dans les Vies.

${ }^{192}$ C'est le titre même de son ouvrage, Il Romanzo greco. Polifonia ed Eros, Venezia, 1989, traduit en français sous le titre moins évocateur de Naissance du roman, ed. du Seuil, 1991. 
était la sienne que rites et théories ne fus sent pas lettre morte, mais réalités vivan tes continuant d'inspirer la morale quoti dienne et les espoirs métaphysiques. La plus belle illustration en est peut-être, comme le suggère L'Érotikos, son union heureuse avec une femme qui écrivit, nous dit-il, un petit traité sur la parure ${ }^{193}$ et qu'il pouvait consoler de la perte de leur petite fille en lui rappelant qu'elle avait, pour ne pas confondre mort et anéantisse ment, "tant le patrios logos que les for mules mystiques du culte de Dionysos dont nous autres initiés nous partageons la connaissance ${ }^{194}$."

Bibl iog raphie

Ba but , D.,

- Plutarque et le stö̈cisme, Paris, 1969.

- "Peinture et dépassement de la réalité dans le Banquet de Platon", REA, 82 (1982) 49-86, repris dans Parerga, CMO n²4, Lyon, 1994, pp. 171-195.

- "Le dialogue de Plutarque Sur le démon de Socrate. Essai d'interpréta tion", B.A.G.B., (1984) 51-76, repris dans Parerga, pp. 405-430.

- "Du scepticisme au dépassement de la raison Philosophie et foi religieuse chez Plutarque", Parerga, Lyon, 1994, pp. 549-581.

Bar ig a zzi, A.,

- "Note critiche ed esegetiche all'Éroticos di Plutarco", Prometheus, 12 (1986) 97-122 et 245-266.

Ca 1 a me, $\mathrm{C}$.,

- L'eros dans la pensée grecque, Paris, Berlin, 1997.
Cant ar el l a, E.,

- Selon la nature, l'usage et la loi. La bisexualité dans le monde antique, trad. fçse, Éd. La Découverte, 1991.

Do v e r, K.,

- Homosexualité grecque, trad. fçse S. Sa ïd, Grenoble, 1982.

Fl a c e l ièr e, R.,

- Plutarque, Euvres morales t. X, Dialogue sur l'Amour, Paris, Les Belles Lettres, 1980.

Fo u c a ul t , M.,

- Histoire de la Sexualité T.III, Le souci de soi, Paris, 1984.

Frazier, F.,

- "Platonisme et Patrios pistis dans le discours central de (chs 13-20)", in A. Pérez Jimenez, J. Garcia Lôpez y Rosa Maria AguiLAR, Plutarco, Platon Aristôteles. Actas del $V$ Congresso Internacional de la 1. P. S. (Madrid-Cuenca, 4-7 de Mayo de 1999), Madrid, 1999, pp. 343-356.

"UÉrotikos et les fragments sur l'a mour de Stobée", in José Ribeiro Ferreira et Delfim Ferreira Leâo (edd.), Os fragmentos de Plutarco e a recepçào da sua obra, Coimbra, 2003, pp. 63-87.

- 'La 'prouesse de Camma' et la fonc tion des exempla dans le Dialogue Sur l'Amour", in A. Pé r e z-Jiméne z $\&$ F. Tit chener (edd.), Historical and Biographical Values of tarch's Works. Studies devoted to Professer Philip Stadter by the Inter national Plutarch Society, 2005, pp. 197-212 (= 2005a)

- “À propos de l’influence de la corné-

193 Praec. conj. 145 A.

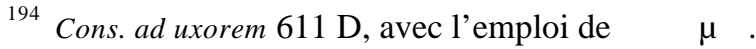


die dans YÉrotikos. Un réexamen de la notion de "dialogue dramatique", in A. Casanova (ed.), Plutarco $e$ l'età ellenistica, Firenze, 2005, pp. 173-205 (= 2005b).

Go essi e r, L.,

- Plutarchs Gedanken über die Ehe, Zurich, 1962.

Go 1 dh il 1 , S.,

- Foucault's Viriginity, Cambridge Univ. Press, 1995.

Ha l pe $r$ in, D.,

- "Plato and Erotic Reciprocity", $\mathrm{Cl}$. Ant., 5 (1986) 60-80.

Hir z e l, R.,

- Der Dialog. Ein literarhistorischer
Versuch,Leipzig, 1895.

Pue ch, B.,

- "Prosopographie des amis de Plutar que", ANRWII. 33. 6, pp. 4831-4893.

Rist , J. M.,

- "Plutarch's Amatorius: A commenta ry on Plato's Theories of Love ?", CQ, 51 (2001)557-575.

Russe I I, D. A.,

- Plutarch, London, 1973.

- "Plutarch. Amatorius 13-18", in J. Moss man (ed.), Plutarch and his Intellectual World, Oxford, 1997, pp. 99-111.

Zunt z, G,

- "Notes on Plutarch's Moralia", RM, (1953) 232-235. 